\title{
Covariant Perturbations of Schwarzschild Black Holes
}

\author{
Chris A. Clarkson \\ Relativity and Cosmology Group, Department of Mathematics and Applied Mathematics, \\ University of Cape Town, Rondebosch 7701, Cape Town, South Africt*
}

Richard K. Barrett

Astronomy and Astrophysics group, Department of Physics and Astronomy, University of Glasgow, University Avenue, Glasgow, G12 8QQ, UK円

(Dated: May 28, 2018)

\begin{abstract}
We present a new covariant and gauge-invariant perturbation formalism for dealing with spacetimes having spherical symmetry (or some preferred spatial direction) in the background, and apply it to the case of gravitational wave propagation in a Schwarzschild black hole spacetime. The 1+3 covariant approach is extended to a ' $1+1+2$ covariant sheet' formalism by introducing a radial unit vector in addition to the timelike congruence, and decomposing all covariant quantities with respect to this. The background Schwarzschild solution is discussed and a covariant characterisation is given. We give the full first-order system of linearised $1+1+2$ covariant equations, and we show how, by introducing (time and spherical) harmonic functions, these may be reduced to a system of first-order ordinary differential equations and algebraic constraints for the $1+1+2$ variables which may be solved straightforwardly. We show how both the odd and even parity perturbations may be unified by the discovery of a covariant, frame- and gauge-invariant, transverse-traceless tensor describing gravitational waves, which satisfies a covariant wave equation equivalent to the ReggeWheeler equation for both even and odd parity perturbations. We show how the Zerilli equation may be derived from this tensor, and derive a similar transverse traceless tensor equivalent to this equation. The so-called 'special' quasinormal modes with purely imaginary frequency emerge naturally. The significance of the degrees of freedom in the choice of the two frame vectors is discussed, and we demonstrate that, for a certain frame choice, the underlying dynamics is governed purely by the Regge-Wheeler tensor. The two transverse-traceless Weyl tensors which carry the curvature of gravitational waves are discussed, and we give the closed system of four first-order ordinary differential equations describing their propagation. Finally, we consider the extension of this work to the study of gravitational waves in other astrophysical situations.
\end{abstract}

\section{INTRODUCTION}

The $1+3$ covariant approach has proven to be an extremely useful technique for developing a detailed understanding of many aspects of relativistic cosmology, both in terms of fully nonlinear GR effects and through the application of the gauge-invariant, covariant perturbation formalism (see [1], for example) to the formation and evolution of density perturbations [2] in the universe and to the physics of the cosmic microwave background [3, 4], amongst other things (see [1] for a comprehensive review). Its strength in cosmological applications lies in the fact that it is well adapted to the system it is describing: all essential information can be captured in a set of $(1+3)$ covariant variables (defined with respect to a preferred timelike observer congruence), that have an immediate physical and geometrical significance. They satisfy a set of evolution and constraint equations, derived from Einstein's field equations, and the Bianchi and Ricci identities, which form a closed system of equations when an equation of state for the matter is chosen. The covariant and gauge-invariant linearisation procedure is easy and transparent: it consists of deciding which variables are 'first order' (or 'of order $\epsilon$ ') and those which are 'zeroth order' - i.e., those which do not vanish in the background, which is usually a Friedmann-Lemaitre-Robertson-Walker (FLRW) model. Products of first-order quantities can then be ignored in the equations. Whenever the background is a homogeneous and isotropic FLRW model, all projected vectors and tensors are first-order, so there is no vector-tensor and tensor-tensor coupling in the equations. Harmonic functions can then be introduced which re-write the equations in scalar form; the resulting system is then in the form of algebraic constraints and some first-order ordinary differential equations; the solution is then straightforward. The key point of the approach is that it deals with physically or geometrically relevant quantities, such as the fractional density gradient, $\mathcal{D}_{a}$, or the electric and magnetic parts of the Weyl tensor, $E_{a b}$ and

*Electronic address: clarkson@maths.uct.ac.za

${ }^{\dagger}$ Electronic address: richard@astro.gla.ac.uk 
$H_{a b}$, respectively, which represent the non-local parts of the gravitational field, and which describe, amongst other things, the propagation of gravitational waves $(\mathrm{GW})$. The variables are also (coordinate) gauge invariant (although there is a frame-gauge freedom in the choice of $u^{a}$ - see below).

The aim of this paper is to extend the gauge-invariant, covariant perturbation formalism to an astrophysical setting. The $1+3$ approach is not appropriate for many situations where such techniques would seem highly desirable: when the spacetime in inhomogeneous, for example, the $1+3$ equations usually become intractable. However, by introducing an additional frame vector, assuming that the background spacetime has some preferred spatial direction (such as in spherically symmetric, or more general locally rotationally symmetric spacetimes, or $G_{2}$ spacetimes) we can in many cases recover all of the advantages of the $1+3$ equations, but in a $1+1+2$ covariant framework [5]. In this paper we introduce the $1+1+2$ formalism, and apply it to linear perturbations of a Schwarzschild spacetime. Not only is this a first step in applying the procedure to more general astrophysical situations (such as perturbations of the interior of compact objects, collapsing and exploding stars, etc.), it also represents an important field of study in itself: with the development of large gravitational wave detectors (e.g., [6]) an improved understanding of the problem of GW propagation around compact objects is certainly timely. The power of the $1+1+2$ technique is clearly shown by the significant results we are able to obtain, relatively simply. For example, we show here that the full description of gravitational waves around a Schwarzschild black hole is governed by closed covariant wave equation, unifying both parities in a single covariantly defined gauge- and frame-invariant transverse-traceless 2-tensor, $W_{a b}$.

Linear perturbations of Schwarzschild black holes have been conventionally studied through perturbations of the metric tensor (or via the Newman-Penrose formalism [7]). In the metric approach, fluctuations of the spacetime geometry are characterised by perturbations in the metric tensor; these fluctuations are determined by closed wave equations - the Regge-Wheeler equation for odd parity perturbations [8] and the Zerilli equation in the even parity regime [9]. These wave equations act on linear combinations of the functions (and their derivatives) appearing in the perturbed metric, but these functions do not determine directly the gravitational waves which they represent; a general coordinate transformation would preserve neither the perturbation functions themselves, nor the wave equations which they satisfy. The approach we develop here is completely covariant, so such issues do not arise. Instead, corresponding wave equations we derive here are formed from covariant and gauge-invariant variables which have a physical significance; furthermore, they do not require a harmonic splitting for their derivation.

The formalism we develop here relies on a further splitting of the spacetime using a radial vector $n^{a}$, in addition to the usual splitting with the timelike vector $u^{a}$ used in the $1+3$ approach. We split the Ricci and Bianchi identities using $u^{a}$ and $n^{a}$ into a coupled set of first order differential equations, plus some constraints. The differential operators we use are along the two vector fields which give us a set of evolution and propagation (along the 'radial' direction) equations, while a derivative formed from a projection orthogonal to $u^{a}$ and $n^{a}$ gives a small number of constraints. The differential equations involve the covariant variables derived from splitting the Weyl tensor (and more generally the Ricci tensor, but this is zero here as we only consider vacuum perturbations), and the kinematics of $u^{a}$ and $n^{a}$. As our background is static and spherically symmetric, we may use harmonic functions for our evolution and projected derivatives, putting our equations into the form of a first order system of ordinary DE's and constraints, which can then be tackled relatively easily.

Previously, 10, 11, 12, 13] (and references therein) developed approaches to stellar and black hole perturbations similar to the method presented here in the sense that they use two orthogonal vectors to form their time and space derivatives. These approaches are fundamentally different from our approach, however, in that they formulate their differential equations as second order PDE's derived from Einstein's field equations (EFE), the solutions of which give the metric functions (as in all metric perturbation approaches). On the other hand, our system of DE's is manifestly first order, as it is derived from the first order Ricci and Bianchi identities ${ }^{1}$, as it involves physical or geometric quantities, and not the metric functions. Second order wave equations may be derived if desired. This is one of the key properties of covariant or tetrad methods. This change in derivative level is conceptually analogous to the change in going from the Lagrangian to Hamiltonian formulations of classical mechanics, from configuration space to phase space.

The layout of the paper is as follows: in the following section we discuss the merits of a $1+1+2$ decomposition of the field equations and set out the $1+1+2$ covariant formalism. Then, in section III we present the full set of $1+1+2$ covariant, gauge-invariant, first-order equations, linearised about a Schwarzschild background and introduce the (spherical and temporal) harmonics on the 'sheet', which enable the equations to be reduced to a set of coupled ODEs for the $1+1+2$ covariant variables. In section IV we prove the existence of a transverse-traceless (TT) tensor that satisfies a closed wave equation equivalent to the Regge-Wheeler equation, valid for harmonics of either parity.

\footnotetext{
${ }^{1}$ We use the Ricci identities applied to our vectors $u^{a}$ and $n^{a}$, so they are second order DE's of $u^{a}$ and $n^{a}$; however, our covariant objects are different projections of $\nabla_{a} u_{b}$ and $\nabla_{a} n_{b}$, so that the Ricci identities are first order DE's in these covariant objects.
} 
Following this we discuss the even parity variable which satisfies a wave equation equivalent to the Zerilli equation; we demonstrate here the existence of an odd parity counterpart, but defer the derivation of the 'Zerilli tensor' until later, Sec. VC Then, in section V we describe in detail the (matrix-based) method of solution of the linear, firstorder system of ODEs for the harmonic components, emphasising the significance of the freedom to choose the frame vectors and showing that with an appropriate choice we can reduce the whole solution for both parities to a single 2-dimensional ODE. The wave equations for the TT electric and magnetic Weyl tensors are also presented, and the closed four-dimensional ODE which they also satisfy is discussed. Finally, we discuss the results we have obtained.

We follow the notation and conventions of 1 ].

\section{THE $1+1+2$ COVARIANT SHEET APPROACH}

Before setting out the principles and equations of the $1+1+2$ covariant formalism it will be illuminating to examine the $1+3$ approach to see where its strengths and weaknesses lie, and why it is so successful in a cosmological setting, but is less useful in other situations, such as in the study of gravitational radiation in a Schwarzschild spacetime which we consider here. In the process we hope to indicate that the $1+1+2$ formalism neatly fills a gap between the $1+3$ and tetrad approaches.

\section{A. 1+3 covariant perturbation theory: why it works in cosmology, but not for black holes}

In a nutshell, the $1+3$ formalism is successful in cosmological applications because the assumed spatial homogeneity and isotropy of the background spacetime means that the only essential coordinate is time: the introduction of an appropriate timelike observer congruence $u^{a}$ allows the full structure of the spacetime to be described solely in terms of ordinary differential equations involving $(1+3)$ scalar quantities because all spatial derivatives, spatial projections of vectors, and projected, symmetric, trace-free parts of tensors must vanish by symmetry.

On the other hand, when the spacetime is not homogeneous and isotropic the resulting equations are not simple ODEs. Consider, for example, a family of static observers around a Schwarzschild black hole, that is, observers on the congruence $u^{a}$ parallel to the timelike conformal Killing vector. Then $u^{a}$ has zero rotation, shear and expansion, but has non-zero acceleration $\dot{u}^{a}$ (reflecting the fact that a force must be applied to prevent infall), and the electric Weyl curvature, $E_{a b}$, is non-zero, while all other covariant quantities are zero. Relative to these observers, $E_{a b}$ measures non-local gravitational effects: in this case the (time-independent) radial tidal forces only, which can be described by a single function of distance from the hole - thanks to the spherical symmetry we can think of it is a tensor describing an essentially scalar phenomenon. The $1+3$ covariant equations describing the spacetime are $^{2}$

$$
\begin{aligned}
\dot{E}_{\langle a b\rangle} & =0, \\
\operatorname{div} \dot{u}+\dot{u}^{2} & =0, \\
\operatorname{curl} \dot{u}_{a} & =0, \\
E_{a b}-\mathrm{D}_{\langle a} \dot{u}_{b\rangle}-\dot{u}_{\langle a} \dot{u}_{b\rangle} & =0, \\
\operatorname{curl} E_{a b}+2 \varepsilon_{c d\langle a} \dot{u}^{c} E_{b\rangle}{ }^{d} & =0, \\
\operatorname{div} E_{a} & =0 .
\end{aligned}
$$

This is already a formidable set of tensor equations; indeed, their solution is basically an intractable problem unless we introduce a full tetrad or revert to a metric based approach. These problems become even more severe when we consider the perturbed spacetime.

In the $1+3$ approach this is achieved by assuming that in general all of the $1+3$ covariant quantities are non-zero, but that any that vanish in the background are small - small enough that we can neglect products of such terms. To get an idea of the horrendous nature of the equations, consider the (gauge-dependent) wave equation for $E_{a b}$, which gives information about gravitational waves (or linear dynamical tidal forces):

$$
\ddot{E}_{\langle a b\rangle}-\mathrm{D}^{2} E_{a b}=-\mathrm{D}^{c} \mathrm{D}_{(a} E_{b) c}+5 \dot{u}^{c} \mathrm{D}_{c} E_{a b}-2 \dot{u}^{c} \mathrm{D}_{\langle a} E_{b\rangle c}-3 E_{c\langle a} \mathrm{D}^{c} \dot{u}_{b\rangle}-E_{c\langle a} \mathrm{D}_{b\rangle} \dot{u}^{c}
$$

\footnotetext{
${ }^{2}$ We use the standard notation whereby a dot represents differentiation along the observers' four-velocity $u^{a}, \dot{\psi}_{a \cdots b} \equiv u^{c} \nabla_{c} \psi_{a \cdots b}$, and $\mathrm{D}_{a}$ is a derivative in the rest space of the observers; $\mathrm{D}_{c} \psi_{a \cdots b} \equiv h_{c}{ }^{d} h_{a}{ }^{e} \cdots h_{b}{ }^{f} \nabla_{d} \psi_{e} \cdots f$, where $h_{a b} \equiv g_{a b}+u_{a} u_{b}$ is the usual projection tensor orthogonal to $u^{a}$. We use angled brackets on indices to donate the projected, symmetric and trace-free part of a tensor.
} 


$$
+\frac{1}{2} \varepsilon_{c d\langle a} \text { curl } \dot{u}^{c} E_{b\rangle}^{d}+2\left(\operatorname{div} \dot{u}+\dot{u}^{2}\right) E_{a b}+3 E_{\langle a}^{c} E_{b\rangle c}-6 \dot{u}^{c} \dot{u}_{\langle a} E_{b\rangle c} .
$$

This also contains information about non-linear tidal forces, which don't propagate at the speed of light, by virtue of the presence of the parts of $E_{a b}$ that do not vanish in the background. But there is no way to separate the two physical effects. In addition, how could we solve this wave equation? In contrast to FLRW models, which have only scalars describing the model after a $1+3$ decomposition, it is the presence of non-zero vectors and tensors in the background spacetime which makes a black hole impossible to deal with in the $1+3$ approach: all the equations have vector-tensor and tensor-tensor coupling in them, rendering them intractable.

The key, then, for the covariant perturbation approach lies in the fact that, in the background, the congruence $u^{a}$ is orthogonal to 3-surfaces of homogeneity and isotropy. In the case of inhomogeneous, spherically symmetric systems, projection via $u^{a}$ is simply not enough. Another vector field is required that is orthogonal to homogeneous and isotropic surfaces. Clearly, after an appropriate projection with $u^{a}$, such surfaces are provided by spheres surrounding the centre of symmetry, and the vector orthogonal to these is a radial vector. We turn now to developing such a formalism.

\section{B. The $1+1+2$ formalism}

In the $1+3$ approach, a timelike threading vector field $u^{a}\left(u^{a} u_{a}=-1\right)$ is introduced, representing the observers' congruence. Given this vector field, the projection tensor $h_{a}{ }^{b}=g_{a}{ }^{b}+u_{a} u^{b}$ is introduced, which projects all vectors and tensors orthogonal to $u^{a}$. Using $h_{a b}$, any 4 -vector may be split into a $\left(1+3\right.$ scalar) part parallel to $u^{a}$ and a (3-vector) part orthogonal to $u^{a}$. Any second rank tensor may be covariantly and irreducibly split into scalar, vector and projected, symmetric, trace-free (PSTF) 3-tensor parts, which requires the alternating tensor $\varepsilon_{a b c}=u^{d} \eta_{d a b c}[1]$. Tensors of higher rank may be similarly split, but are rarely used (an important exception being CMB physics [3, 4]). These are the fundamental quantities describing the spacetime, after the introduction of $u^{a}$.

We now introduce another vector field and perform another split, but this time of the $1+3$ equations. The ' $1+1+2$ ' decomposition we develop here has been partially studied before, mostly in the context of symmetries of solutions of the EFE [5, 14, 15]. It was introduced by [16] and further developed in [5, 17]. However, there are importances differences with the work presented here. In the following we assume the $1+3$ covariant split of the equations (as given in [1], for example), with all tensors split into scalars, vectors and PSTF tensors with respect to $u^{a}$.

Take a unit vector $n^{a}$ orthogonal to $u^{a}: n^{a} n_{a}=1, u^{a} n_{a}=0$, and define the projection tensor

$$
N_{a}^{b} \equiv h_{a}^{b}-n_{a} n^{b}=g_{a}^{b}+u_{a} u^{b}-n_{a} n^{b},
$$

which projects vectors orthogonal to $n^{a}$ (and $u^{a}$ ): $n^{a} N_{a b}=0=u^{a} N_{a b}$, onto 2-surfaces $\left(N_{a}^{a}=2\right)$ which we refer to as the 'sheet' (to carry the sewing analogy of the threading approach into the realm of the ridiculous).

Any 3 -vector $\psi^{a}$ can now be irreducibly split into a scalar, $\Psi$, which is the part of the vector parallel to $n^{a}$, and a vector, $\Psi^{a}$, lying in the sheet orthogonal to $n^{a}$;

$$
\psi^{a}=\Psi n^{a}+\Psi^{a}, \quad \text { where } \Psi \equiv \psi_{a} n^{a}, \quad \text { and } \quad \Psi^{a} \equiv N^{a b} \psi_{b} \equiv \psi^{\bar{a}},
$$

where we use a bar over an index to denote projection with $N_{a b}$. Similarly, any PSTF tensor, $\psi_{a b}$, can now be split into scalar, vector and tensor (which are PSTF with respect to $n^{a}$ ) parts:

$$
\psi_{a b}=\psi_{\langle a b\rangle}=\Psi\left(n_{a} n_{b}-\frac{1}{2} N_{a b}\right)+2 \Psi_{(a} n_{b)}+\Psi_{a b},
$$

where

$$
\begin{aligned}
\Psi & \equiv n^{a} n^{b} \psi_{a b}=-N^{a b} \psi_{a b}, \\
\Psi_{a} & \equiv N_{a}^{b} n^{c} \psi_{b c}=\Psi_{\bar{a}}, \\
\Psi_{a b} & \equiv \psi_{\{a b\}} \equiv\left(N_{(a}{ }^{c} N_{b)}{ }^{d}-\frac{1}{2} N_{a b} N^{c d}\right) \psi_{c d} .
\end{aligned}
$$

We use curly brackets to denote the PSTF with respect to $n^{a}$ part of a tensor. Note that for 2nd-rank tensors in the 1+1+2 formalism 'PSTF' is precisely equivalent to 'transverse-traceless'. ${ }^{3}$ Note also that $h_{\{a b\}}=0, N_{\langle a b\rangle}=$ $-n_{\langle a} n_{b\rangle}=N_{a b}-\frac{2}{3} h_{a b}$.

\footnotetext{
${ }^{3}$ Our use of the term 'transverse' only refers to the fact that the tensor is orthogonal to $n^{a}$; this does not imply it is divergence free, which is an additional property of tensors in the commonly used TT gauge of the plane wave approximation.
} 
We also define the alternating Levi-Civita 2-tensor

$$
\varepsilon_{a b} \equiv \varepsilon_{a b c} n^{c}=u^{d} \eta_{d a b c} n^{c},
$$

so that $\varepsilon_{a b} n^{b}=0=\varepsilon_{(a b)}, \quad \varepsilon_{a b c}=n_{a} \varepsilon_{b c}-n_{b} \varepsilon_{a c}+n_{c} \varepsilon_{a b}, \varepsilon_{a b} \varepsilon^{c d}=N_{a}{ }^{c} N_{b}{ }^{d}-N_{a}{ }^{d} N_{b}{ }^{c}, \varepsilon_{a}{ }^{c} \varepsilon_{b c}=N_{a b}$, and $\varepsilon^{a b} \varepsilon_{a b}=2$. Note that for a 2-vector $\Psi^{a}, \varepsilon_{a b}$ may be used to form a vector orthogonal to $\Psi^{a}$ but of the same length.

With these definitions, then, we may split any object into scalars, 2-vectors in the sheet, and transverse-traceless 2-tensors, also defined in the sheet. These three types of objects are the only objects which appear, after a complete splitting. Hereafter, we will assume such a split has been made, and 'vector' will generally refer to a vector projected orthogonal to $u^{a}$ and $n^{a}$, and 'tensor' will generally mean transverse-traceless tensor, defined by Eq. (11).

There are two new derivatives of interest now, which $n^{a}$ defines, for any object $\psi \ldots \ldots$ :

$$
\begin{aligned}
\hat{\psi}_{a \cdots b}{ }^{c \cdots d} & \equiv n^{e} \mathrm{D}_{e} \psi_{a \cdots b}{ }^{c \cdots d}, \\
\delta_{e} \psi_{a \cdots b}{ }^{c \cdots d} & \equiv N_{e}{ }^{j} N_{a}{ }^{f} \cdots N_{b}{ }^{g} N_{h}{ }^{c} \cdots N_{i}{ }^{d} \mathrm{D}_{j} \psi_{f \cdots g}{ }^{h \cdots i} .
\end{aligned}
$$

The hat-derivative is the derivative along the vector field $n^{a}$ in the surfaces orthogonal to $u^{a}$. This definition represents a conceptual divergence from the tetrad approach, in which the basis vectors appear on an equal footing [i.e., with $\nabla_{a}$ rather than $\mathrm{D}_{a}$ in Eq. (13)]. As a result, the congruence $u^{a}$ retains the primary importance it has in the $1+3$ covariant approach. (We choose to think of $\mathcal{A} \equiv u^{a} n^{b} \nabla_{a} u_{b}=-u^{a} u^{b} \nabla_{a} n_{b}$ as the radial component of the acceleration of $u^{a}$, rather than the time component of $\dot{n}^{a}$.) The $\delta$-derivative, defined by Eq. (14) is a projected derivative on the sheet, with projection on every free index.

With these definitions we may now decompose the covariant derivative of $n^{a}$ orthogonal to $u^{a}$ :

$$
\mathrm{D}_{a} n_{b}=n_{a} a_{b}+\frac{1}{2} \phi N_{a b}+\xi \varepsilon_{a b}+\zeta_{a b},
$$

where

$$
\begin{aligned}
a_{a} & \equiv n^{c} \mathrm{D}_{c} n_{a}=\hat{n}_{a}, \\
\phi & \equiv \delta_{a} n^{a}, \\
\xi & \equiv \frac{1}{2} \varepsilon^{a b} \delta_{a} n_{b}, \\
\zeta_{a b} & \equiv \delta_{\{a} n_{b\}} .
\end{aligned}
$$

We may interpret these as follows: travelling along $n^{a}, \phi$ represents the sheet expansion, $\zeta_{a b}$ is the shear of $n^{a}$ (distortion of the sheet), and $a^{a}$ its acceleration, while $\xi$ represents a 'twisting' of the sheet - the rotation of $n^{a}$ [17]. The other derivative of $n^{a}$ is its change along $u^{a}$,

$$
\dot{n}_{a}=\mathcal{A} u_{a}+\alpha_{a} \quad \text { where } \quad \alpha_{a} \equiv \dot{n}_{\bar{a}} \quad \text { and } \quad \mathcal{A}=n^{a} \dot{u}_{a} .
$$

The new variables $a_{a}, \phi, \xi, \zeta_{a b}$ and $\alpha_{a}$ are fundamental objects in the spacetime, and their dynamics gives us information about the spacetime geometry. They are treated on the same footing as the kinematical variables of $u^{a}$ in the $1+3$ approach (which also appear here).

Note that for a scalar, we have $\mathrm{D}_{a} \Psi=\hat{\Psi} n_{a}+\delta_{a} \Psi$, while for any vector $\Psi^{a}$ orthogonal to $n^{a}$ and $u^{a}$ (i.e., $\Psi^{a}=\Psi^{\bar{a}}$ ), we may decompose the different parts of its spatial derivative:

$$
\mathrm{D}_{a} \Psi_{b}=-n_{a} n_{b} \Psi_{c} a^{c}+n_{a} \hat{\Psi}_{\bar{b}}-n_{b}\left[\frac{1}{2} \phi \Psi_{a}+\left(\xi \varepsilon_{a c}+\zeta_{a c}\right) \Psi^{c}\right]+\delta_{a} \Psi_{b}
$$

Similarly, for a tensor $\Psi_{a b}: \Psi_{a b}=\Psi_{\{a b\}}$, we have

$$
\mathrm{D}_{a} \Psi_{b c}=-2 n_{a} n_{(b} \Psi_{c) d} a^{d}+n_{a} \hat{\Psi}_{b c}-2 n_{(b}\left[\frac{1}{2} \phi \Psi_{c) a}+\Psi_{c)}{ }^{d}\left(\xi \varepsilon_{a d}+\zeta_{a d}\right)\right]+\delta_{a} \Psi_{b c} .
$$

Note that $\dot{N}_{a b}=2 u_{(a} \dot{u}_{b)}-2 n_{(a} \dot{n}_{b)}, \quad \hat{N}_{a b}=-2 n_{(a} a_{b)}, \quad \delta_{c} N_{a b}=0$; while $\dot{\varepsilon}_{a b}=-2 u_{[a} \varepsilon_{b] c} \mathcal{A}^{c}+2 n_{[a} \varepsilon_{b] c} \alpha^{c}, \hat{\varepsilon}_{a b}=$ $2 n_{[a} \varepsilon_{b] c} a^{c}, \delta_{c} \varepsilon_{a b}=0$.

We take $n^{a}$ to be arbitrary at this point, and then split the usual $1+3$ kinematical and Weyl quantities into the irreducible set $\left\{\theta, \mathcal{A}, \Omega, \Sigma, \mathcal{E}, \mathcal{H}, \mathcal{A}^{a}, \Sigma^{a}, \mathcal{E}^{a}, \mathcal{H}^{a}, \Sigma_{a b}, \mathcal{E}_{a b}, \mathcal{H}_{a b}\right\}$ using (9) and (10) as follows:

$$
\begin{aligned}
\dot{u}^{a} & =\mathcal{A} n^{a}+\mathcal{A}^{a} \\
\omega^{a} & =\Omega n^{a}+\Omega^{a} \\
\sigma_{a b} & =\Sigma\left(n_{a} n_{b}-\frac{1}{2} N_{a b}\right)+2 \Sigma_{(a} n_{b)}+\Sigma_{a b}, \\
E_{a b} & =\mathcal{E}\left(n_{a} n_{b}-\frac{1}{2} N_{a b}\right)+2 \mathcal{E}_{(a} n_{b)}+\mathcal{E}_{a b}, \\
H_{a b} & =\mathcal{H}\left(n_{a} n_{b}-\frac{1}{2} N_{a b}\right)+2 \mathcal{H}_{(a} n_{b)}+\mathcal{H}_{a b} .
\end{aligned}
$$

Having described the splitting of the $1+3$ variables to obtain their $1+1+2$ parts, and the introduction of the new $1+1+2$ variables corresponding to the irreducible parts of $\nabla_{a} n_{b}$, it only remains to apply this splitting procedure to the $1+3$ equations themselves. We give these equations in section $\amalg$ linearised about a Schwarzschild background. 


\section{The Ricci identities}

Once the vector $n^{a}$ has been introduced it is possible, and necessary, to augment the $1+3$ equations with the Ricci identities for $n^{a}$; without these we do not have enough equations to determine the new $1+1+2$ variables. The Ricci identities for $n^{a}$ are

$$
R_{a b c} \equiv 2 \nabla_{[a} \nabla_{b]} n_{c}-R_{a b c d} n^{d}=0
$$

where $R_{a b c d}$ is the Riemann curvature tensor. This 3 -index tensor may be covariantly split using the two vector fields $u^{a}$ and $n^{a}$, and gives dynamical equations for the covariant parts of the derivative of $n^{a}$ (namely $\alpha_{a}, a_{a}, \phi, \xi$ and $\zeta_{a b}$ ) in the form of evolution equations, involving dot-derivatives of these variables, and propagation equations, involving hat-derivatives. In order to facilitate the calculation of these Ricci identities, which appear in the following section, we give here the expression for the full covariant derivative of $n^{a}$ in terms of the relevant $1+1+2$ variables:

$$
\nabla_{a} n_{b}=-\mathcal{A} u_{a} u_{b}-u_{a} \alpha_{b}+\left(\Sigma+\frac{1}{3} \theta\right) n_{a} u_{b}+\left[\Sigma_{a}-\varepsilon_{a c} \Omega^{c}\right] u_{b}+n_{a} a_{b}+\frac{1}{2} \phi N_{a b}+\xi \varepsilon_{a b}+\zeta_{a b},
$$

which may be inserted into Eq. (28).

\section{Commutation relations}

In general the three derivatives we now have defined, '. ', '^ ' and ' $\delta_{a}$ ' do not commute. Instead, when acting on a scalar $\psi$, they satisfy:

$$
\begin{aligned}
\hat{\dot{\psi}}-\dot{\hat{\psi}} & =-\dot{\psi} \mathcal{A}+\left(\frac{1}{3} \theta+\Sigma\right) \hat{\psi}+\left(\Sigma_{a}+\varepsilon_{a b} \Omega^{b}-\alpha_{a}\right) \delta^{a} \psi, \\
\delta_{a} \dot{\psi}-N_{a}^{b}\left(\delta_{b} \psi\right)^{\cdot} & =-\mathcal{A}_{a} \dot{\psi}+\left(\alpha_{a}+\Sigma_{a}-\varepsilon_{a b} \Omega^{b}\right) \hat{\psi}+\left(\frac{1}{3} \theta-\frac{1}{2} \Sigma\right) \delta_{a} \psi+\left(\Sigma_{a b}+\Omega \varepsilon_{a b}\right) \delta^{b} \psi \\
\delta_{a} \hat{\psi}-N_{a}{ }^{b} \widehat{\left(\delta_{b} \psi\right)} & =-2 \varepsilon_{a b} \Omega^{b} \dot{\psi}+a_{a} \hat{\psi}+\frac{1}{2} \phi \delta_{a} \psi+\left(\zeta_{a b}+\xi \varepsilon_{a b}\right) \delta^{b} \psi \\
\delta_{a} \delta_{b} \psi-\delta_{b} \delta_{a} \psi & =2 \varepsilon_{a b}(\Omega \dot{\psi}-\xi \hat{\psi})+2 a_{[a} \delta_{b]} \psi .
\end{aligned}
$$

These last two equations are the decomposition of the $1+3$ commutation relation

$$
\operatorname{curl} \mathrm{D}_{a} \psi=2 \dot{\psi} \omega_{a}
$$

These relations are considerably more complicated for vectors and tensors.

From Eq. (33), we see that our sheet will be a genuine 2-surface in the spacetime (and, in particular, that the derivative $\delta_{a}$ will be a true covariant derivative on this surface) if and only if $\xi=\Omega=a^{a}=0$. (Recall that the $1+3$ spatial metric $h_{a b}$ corresponds to a genuine 3 -surface when $\omega^{a}=0$.) Otherwise, the sheet is really just a collection of tangent planes. In addition, the two vectors $u^{a}$ and $n^{a}$ are 2-surface forming if and only if the commutator $[u, n]$ in (30) has no component in the sheet: that is, when Greenberg's vector, $\Sigma^{a}+\varepsilon^{a b} \Omega_{b}-\alpha^{a}$, vanishes [14] - see Eq. (30).

\section{PERTURBATIONS AROUND A SCHWARZSCHILD BLACK HOLE}

For an exact Schwarzschild black hole it turns out the the only non-zero $1+1+2$ variables are the scalars $\{\mathcal{A}, \mathcal{E}, \phi\}$ (and their derivatives $\{\hat{\mathcal{A}}, \hat{\mathcal{E}}, \hat{\phi}\}$ ), a covariant characterisation of the Schwarzschild solution. (We saw in section that $\mathcal{A}$ and $\mathcal{E}$ are the only non-zero parts of the $1+3$ variables, and it is clear by considering Gauss' theorem that $\phi$, the divergence of $n^{a}$, must also be non-zero. We will consider the background solution in more detail in section IIIB) Because the background solution involves only scalars, under any perturbation all vectors and tensors are first-order, which greatly simplifies things, as we discussed in section ПA

The usual $1+3$ evolution and constraint equations may be further split with the vector $n^{a}$, into a set of evolution (along $u^{a}$ ) and propagation (along $n^{a}$ ) equations. Together with the Ricci identities for $n^{a}$, we find a complete set of propagation, evolution and constraint equations - the constraints being those equations with no hat- or dot-derivatives in them. We will give the complete nonlinear equations elsewhere, as they are large and unpleasant. Here, however, we will give the vacuum equations linearised around a Schwarzschild black hole background. Our linearisation procedure is straightforward: as in the $1+3$ approach we neglect all products of first-order quantities; first-order quantities being those which vanish in the background:

$$
\left\{\theta, \Omega, \Sigma, \xi, \mathcal{H}, \alpha^{a}, a^{a}, \mathcal{A}^{a}, \Omega^{a}, \Sigma^{a}, \mathcal{E}^{a}, \mathcal{H}^{a}, \zeta_{a b}, \Sigma_{a b}, \mathcal{E}_{a b}, \mathcal{H}_{a b}\right\}=\mathcal{O}(\epsilon)
$$


(along with their derivatives, and dot- and $\delta$-derivatives of $\{\mathcal{A}, \mathcal{E}, \phi\}$ ), where $\epsilon$ is a 'smallness' parameter, which measures departures from an exact black hole. So, for example, one could define $\epsilon \simeq \Omega / \phi$, or $\epsilon \simeq \sqrt{\mathcal{E}_{a b} \mathcal{E}^{a b}} / \mathcal{E}$, and so on. From now on all equations are linearised about a Schwarzschild black hole, and equations of the form $A=B$ generally mean $A-B=\mathcal{O}\left(\epsilon^{2}\right)$ (in keeping with usual practice, we will not distinguish between this and real equality).

When studying cosmological perturbations using the $1+3$ approach, the evolution equations are of prime importance, since time is the only remaining essential parameter: the goal is to find the evolution of seed perturbations corresponding to the various spatial harmonics. In contrast, for the black hole perturbations analysed here the time invariance and spherical symmetry of the background mean that radius is the interesting parameter, and so the propagation equations are the key: we want to find the variation with radius of the various (time and spherical) harmonic components. Thus, we present the propagation (hat) equations first, and relegate the evolution equations to a secondary position. This will be helpful when we come to solve the equations in Section $\mathrm{V}$

Propagation ${ }^{4}$ :

$$
\begin{aligned}
\hat{\phi} & =-\frac{1}{2} \phi^{2}-\mathcal{E}+\delta_{a} a^{a} \\
\hat{\xi} & =-\phi \xi+\frac{1}{2} \varepsilon_{a b} \delta^{a} a^{b} \\
\hat{\alpha}_{\bar{a}}-\dot{a}_{\bar{a}} & =-\left(\frac{1}{2} \phi+\mathcal{A}\right) \alpha_{a}+\left(\frac{1}{2} \phi-\mathcal{A}\right)\left(\Sigma_{a}+\varepsilon_{a b} \Omega^{b}\right)-\varepsilon_{a b} \mathcal{H}^{b} \\
\frac{2}{3} \hat{\theta}-\hat{\Sigma} & =\frac{3}{2} \phi \Sigma+\delta_{a} \Sigma^{a}+\varepsilon_{a b} \delta^{a} \Omega^{b} \\
\hat{\mathcal{A}}-\dot{\theta} & =-\mathcal{A}(\phi+\mathcal{A})-\delta_{a} \mathcal{A}^{a} \\
\hat{\Omega} & =(\mathcal{A}-\phi) \Omega-\delta_{a} \Omega^{a} \\
\hat{\mathcal{E}} & =-\frac{3}{2} \phi \mathcal{E}-\delta_{a} \mathcal{E}^{a} \\
\hat{\mathcal{H}} & =-\frac{3}{2} \phi \mathcal{H}-\delta_{a} \mathcal{H}^{a}-3 \mathcal{E} \Omega \\
\hat{\Sigma}_{\bar{a}}-\varepsilon_{a b} \hat{\Omega}^{b} & =-\frac{3}{2} \phi \Sigma_{a}+\left(2 \mathcal{A}+\frac{1}{2} \phi\right) \varepsilon_{a b} \Omega^{b}+\frac{2}{3} \delta_{a} \theta+\frac{1}{2} \delta_{a} \Sigma-\varepsilon_{a b} \delta^{b} \Omega-\delta^{b} \Sigma_{a b}, \\
\hat{\mathcal{A}}_{\bar{a}}-2 \dot{\Sigma}_{\bar{a}} & =2 \mathcal{E}_{a}-\mathcal{A} a_{a}-\delta_{a} \mathcal{A}-2\left(\mathcal{A}-\frac{1}{4} \phi\right) \mathcal{A}_{a}, \\
\hat{\mathcal{E}}_{\bar{a}} & =-\frac{3}{2} \phi \mathcal{E}_{a}+\frac{1}{2} \delta_{a} \mathcal{E}-\frac{3}{2} \mathcal{E} a_{a}-\delta^{b} \mathcal{E}_{a b}, \\
\hat{\mathcal{H}}_{\bar{a}} & =-\frac{3}{2} \phi \mathcal{H}_{a}+\frac{1}{2} \delta_{a} \mathcal{H}-\delta^{b} \mathcal{H}_{a b}+\frac{3}{2} \mathcal{E}\left(\Omega_{a}-\varepsilon_{a b} \Sigma^{b}\right) \\
\hat{\Sigma}_{\{a b\}} & =-\frac{1}{2} \phi \Sigma_{a b}+\delta_{\{a} \Sigma_{b\}}-\varepsilon_{c\{a} \delta^{c} \Omega_{b\}}-\varepsilon_{c\{a} \mathcal{H}_{b\}}{ }^{c} \\
\hat{\zeta}_{\{a b\}} & =-\phi \zeta_{a b}-\mathcal{E}_{a b}+\delta_{\{a} a_{b\}}, \\
\dot{\mathcal{E}}_{\{a b\}}-\varepsilon_{c\{a} \hat{\mathcal{H}}_{b\}}^{c} & =-\frac{3}{2} \mathcal{E} \Sigma_{a b}+\varepsilon_{c\{a}\left(-\delta^{c} \mathcal{H}_{b\}}+\left(\frac{1}{2} \phi+2 \mathcal{A}\right) \mathcal{H}_{b\}}{ }^{c}\right) \\
\dot{\mathcal{H}}_{\{a b\}}+\varepsilon_{c\{a} \hat{\mathcal{E}}_{b\}}^{c} & =\varepsilon_{c\{a}\left(\delta^{c} \mathcal{E}_{b\}}+\frac{3}{2} \mathcal{E} \zeta_{b\}}{ }^{c}-\left(\frac{1}{2} \phi+2 \mathcal{A}\right) \mathcal{E}_{b\}}{ }^{c}\right)
\end{aligned}
$$

Evolution ${ }^{5}$ :

$$
\begin{aligned}
\dot{\phi} & =\left(\frac{1}{2} \phi-\mathcal{A}\right)\left(\Sigma-\frac{2}{3} \theta\right)+\delta_{a} \alpha^{a}, \\
\dot{\xi} & =-\left(\frac{1}{2} \phi-\mathcal{A}\right) \Omega+\frac{1}{2} \mathcal{H}+\frac{1}{2} \varepsilon_{a b} \delta^{a} \alpha^{b}, \\
\dot{\Omega} & =\xi \mathcal{A}+\frac{1}{2} \varepsilon_{a b} \delta^{a} \mathcal{A}^{b} \\
\frac{2}{3} \dot{\theta}-\dot{\Sigma} & =\mathcal{E}+\phi \mathcal{A}+\delta_{a} \mathcal{A}^{a} \\
\dot{\mathcal{E}} & =\left(\frac{3}{2} \Sigma-\theta\right) \mathcal{E}+\varepsilon_{a b} \delta^{a} \mathcal{H}^{b}
\end{aligned}
$$

\footnotetext{
4 These equations are derived as follows: Eq. (36) from $n^{a} N^{b c} R_{a b c}$; Eq. (37) from $n^{a} \varepsilon^{b c} R_{a b c}$; Eq. (38) from $u^{a} n^{b} R_{a b \bar{c}}=n^{a} u^{b} R_{a b \bar{c}}$; Eqs. (39) and 44) from the shear divergence equation, $\left(C_{1}\right)^{a}$; Eq. 40 from the Raychaudhuri equation; Eq. (41) from the rotation divergence equation, $C_{2}$; Eqs. 42 and (46) from the electric Weyl divergence equation, $\left(C_{4}\right)^{a}$; Eqs. 43) and 47 from the magnetic Weyl divergence equation, $\left(C_{5}\right)^{a}$; Eq. 45 from the shear evolution equation; Eq. 48 from the $H_{a b}$-equation, $\left(C_{3}\right)_{\{a b\}}$; Eq. 49 from $n^{a} R_{a\{b c\}}$; Eq. [50] from the electric Weyl evolution equation; and Eq. (51) from the magnetic Weyl evolution equation.

5 These are derived as follows: Eq. (52) from $u^{a} N^{b c} R_{a b c}$; Eq. (53) from $u^{a} \varepsilon^{b c} R_{a b c}$; Eq. (54) from the rotation evolution equation; Eq. (55) from $u^{a} n^{b} u^{c} R_{a b c}=-n^{a} u^{b} u^{c} R_{a b c}$ and the Raychaudhuri equation; Eqs. [56) and [59] from the electric Weyl evolution equation [59] also uses [47]; Eqs. (57) and [60) from the magnetic Weyl evolution equation [60) also uses [46]; Eq. (58) from the rotation and shear evolution equations; Eq. (61) from the shear evolution equation; and Eq. 62 from $u^{c} R_{c\{a b\}}$.
} 


$$
\begin{aligned}
\dot{\mathcal{H}} & =-3 \mathcal{E} \xi-\varepsilon_{a b} \delta^{a} \mathcal{E}^{b} \\
\dot{\Sigma}_{\bar{a}}-\varepsilon_{a b} \dot{\Omega}^{b} & =-\mathcal{E}_{a}+\delta_{a} \mathcal{A}+\left(\mathcal{A}-\frac{1}{2} \phi\right) \mathcal{A}_{a}, \\
\dot{\mathcal{E}}_{\bar{a}} & =-\frac{3}{2} \mathcal{E} \alpha_{a}+\frac{1}{2} \varepsilon_{a b}\left(\delta^{b} \mathcal{H}+(\phi-2 \mathcal{A}) \mathcal{H}^{b}\right)-\varepsilon_{c\{d} \delta^{d} \mathcal{H}_{a\}}{ }^{c}, \\
\dot{\mathcal{H}}_{\bar{a}} & =-\frac{3}{2} \mathcal{E} \varepsilon_{a b} \mathcal{A}^{b}-\frac{1}{2} \varepsilon_{a b}\left(\delta^{b} \mathcal{E}+(\phi-2 \mathcal{A}) \mathcal{E}^{b}\right)+\varepsilon_{c\{d} \delta^{d} \mathcal{E}_{a\}}{ }_{a\}}, \\
\dot{\Sigma}_{\{a b\}} & =\mathcal{A} \zeta_{a b}-\mathcal{E}_{a b}+\delta_{\{a} \mathcal{A}_{b\}}, \\
\dot{\zeta}_{\{a b\}} & =-\left(\frac{1}{2} \phi-\mathcal{A}\right) \Sigma_{a b}-\varepsilon_{c\{a} \mathcal{H}_{b\}}{ }^{c}+\delta_{\{a} \alpha_{b\}} .
\end{aligned}
$$

Constraint ${ }^{6}$ :

$$
\begin{aligned}
\delta_{a} \Sigma-\frac{2}{3} \delta_{a} \theta+2 \varepsilon_{a b} \delta^{b} \Omega+2 \delta^{b} \Sigma_{a b} & =-\phi \Sigma_{a}+\phi \varepsilon_{a b} \Omega^{b}-2 \varepsilon_{a b} \mathcal{H}^{b}, \\
\delta_{a} \Omega^{a}+\varepsilon^{a b} \delta_{a} \Sigma_{b} & =(2 \mathcal{A}-\phi) \Omega+\mathcal{H}, \\
\frac{1}{2} \delta_{a} \phi-\varepsilon_{a b} \delta^{b} \xi-\delta^{b} \zeta_{a b} & =-\mathcal{E}_{a} .
\end{aligned}
$$

These equations must of course be consistent with one another: the constraints must evolve and propagate consistently, and each first-order variable must satisfy the commutation relation

$$
\mathcal{C}[\Psi] \equiv \dot{\hat{\Psi}}-\hat{\dot{\Psi}}-\mathcal{A} \dot{\Psi}=0,
$$

while the background scalars must satisfy the commutation relation (30). It turns out after an arduous calculation that all the equations are consistent.

\section{A. Frame choice}

In this work we are presenting a gauge invariant, covariant approach to perturbations of spherically symmetric spacetimes, based on the introduction of a partial frame, that is, of two basis vectors. In GR there are two 'gauge' freedoms: the choice of coordinates and the freedom to choose a frame basis in the tangent space at each point. These have their direct analogues in perturbation theory, where we imagine that the true spacetime we are studying is "close to' some given, idealised background spacetime. Although not formally the same thing, choosing a coordinate system in the true spacetime is in practice equivalent to fixing the mapping between the true and background spacetimes allowing the direct comparison of scalar, vector and tensor objects in the two spacetimes at corresponding points (see [18]). In metric-based (non-covariant) approaches to the perturbation problem the first step is to find a nice coordinate system in the true spacetime, corresponding to that in the background, and to write equations for the derivatives of the perturbations of scalars, vectors and tensors with respect to these coordinates.

In covariant (partial-)frame approaches, on the other hand, one tries to avoid explicit reference to the background, using it merely to determine which quantities are zeroth order (i.e., which do not vanish in the background). Given the frame vectors, a set of covariant (that is, coordinate invariant) equations describing the true spacetime are written down. (Coordinate-)gauge invariance will hold, according to the Stewart-Walker lemma [19], if we can find a complete set of variables all of which vanish in the background. However, since the covariant variables are the projections of tensors with respect to the frame vectors and the projected parts of the of frame-vector covariant derivatives, the equations and their solutions will, in general, depend on the particular choice of frame vectors. However, since the true spacetime lacks the symmetry of the background, there is, in general, no unique covariant definition of the frame vectors, and one can always perform a first-order rotation of these. This freedom can easily be seen in the preceding set of equations: there are no evolution equations for $\mathcal{A}, \mathcal{A}_{a}$, and $\alpha_{a}$, while there is no propagation equation for $a_{a}$. Indeed, this is true in any spacetime, as one can choose the frame vectors at any point freely, the motion of which must be put into the equations by hand (GR can't predict this!).

In what follows we will reserve the term 'gauge invariant' to refer to the invariance of the equations under the mapping between the true and background spacetimes (in the sense of the Stewart-Walker lemma), and will use 'frame invariant' to describe invariance under the choice of frame vectors. Our $1+1+2$ formalism, in common with the $1+3$ and Newman-Penrose approaches, is gauge invariant, but not frame invariant.

\footnotetext{
${ }^{6}$ These are derived as follows: Eq. [63) from $\left(C_{3}\right)_{a b} n^{b}$ and $\left(C_{1}\right)_{\bar{a}}$, or $n^{a} u^{c} R_{a \bar{b} c}$; Eq. [64) from $\varepsilon^{a b} u^{c} R_{a b c}$; and Eq. [65 from $N^{b c} R_{\bar{a} b c}$. Note that the equation formed from $\left(C_{3}\right)_{a b} n^{a} n^{b}$ is equivalent to Eqs (41) and (64).
} 
The equations as they are presented above are completely general, involving no particular choice of either frame vector. Given a timelike congruence $u^{a}$, obvious choices are possible: for example, we could take $n^{a}$ to be parallel to the acceleration $\dot{u}^{a}$, leading to the frame condition $\mathcal{A}^{a}=0$, a physically plausible choice for observers hovering above the black hole as one could always dexterously align ones rocket to make this so (objects dropped onto the floor of the rocket would fall directly 'down'); or we could demand that $n^{a}$ be parallel to the 'radial' eigenvector of the electric Weyl tensor $E_{a b}$, which leads to the frame choice $\mathcal{E}^{a}=0$. Indeed, we can set any 2 -vector to zero by these considerations. We shall not impose a frame condition at the moment, however, as there is no need.

\section{B. Background Solutions}

In the background we have, by setting all vectors, tensors and time derivatives to zero and retaining only the zeroth-order scalars:

$$
\begin{aligned}
& \hat{\phi}=-\frac{1}{2} \phi^{2}-\mathcal{E}, \\
& \hat{\mathcal{E}}=-\frac{3}{2} \phi \mathcal{E}
\end{aligned}
$$

together with

$$
\mathcal{E}+\mathcal{A} \phi=0
$$

which is Eq. (55). Note that these three equations are sufficient: Eq. (40) is satisfied.

If we associate our hat-derivative with an affine parameter $\rho$, i.e., ${ }^{\wedge}=d / d \rho$, then we may solve these equations. The parametric solutions to our background equations are, in terms of either a parameter $x$ or $r$,

$$
\begin{aligned}
\mathcal{E} & =-\frac{1}{(2 m)^{2}} \operatorname{sech}^{6} x=-\frac{2 m}{r^{3}} \\
\phi & =\frac{1}{m} \sinh x \operatorname{sech}^{3} x=\frac{2}{r} \sqrt{1-\frac{2 m}{r}} \\
\mathcal{A} & =\frac{1}{4 m} \operatorname{cosech} x \operatorname{sech}^{3} x=\frac{m}{r^{2}}\left(1-\frac{2 m}{r}\right)^{-1 / 2} ;
\end{aligned}
$$

where

$$
\rho=2 m\{x+\sinh x \cosh x\},
$$

and the usual Schwarzschild coordinate

$$
r=2 m \cosh ^{2} x
$$

These form a one-parameter family of solutions, parameterised by the constant $m$, which is just the Schwarzschild mass. The Schwarzschild solution is given for $2 m<r<\infty$ for $0<x<\infty$; the interior solution may be found by this approach, but we will not require it here.

We show a plot of $\phi$ and $\mathcal{A}$ with $r$ in Fig. 11 This shows how the expansion of $n^{a}$ starts from zero at the horizon, is largest at the photon sphere, before dropping to zero again as $r \rightarrow \infty$.

The solutions given by (72) represent the general solution to the system of equations (67), (68) and (69), which are the covariant $1+1+2$ equations under the conditions of the spacetime being static, spherically symmetric and vacuum; hence, we may covariantly characterise the Schwarzschild solution by $\{\mathcal{A}, \mathcal{E}, \phi\} \neq 0$, with all other covariant quantities being zero. ${ }^{7}$

\section{Gauge invariant variables}

We have developed a set of covariant equations describing a perturbed black hole, with all variables defined with respect to a family of observers $u^{a}$ with a preferred radial vector field $n^{a}$, which these observers can choose; firstorder variables have a clear physical or geometrical meaning. However, not all variables appearing in the equations

\footnotetext{
7 We would like to thank an anonymous referee for drawing this to our attention.
} 


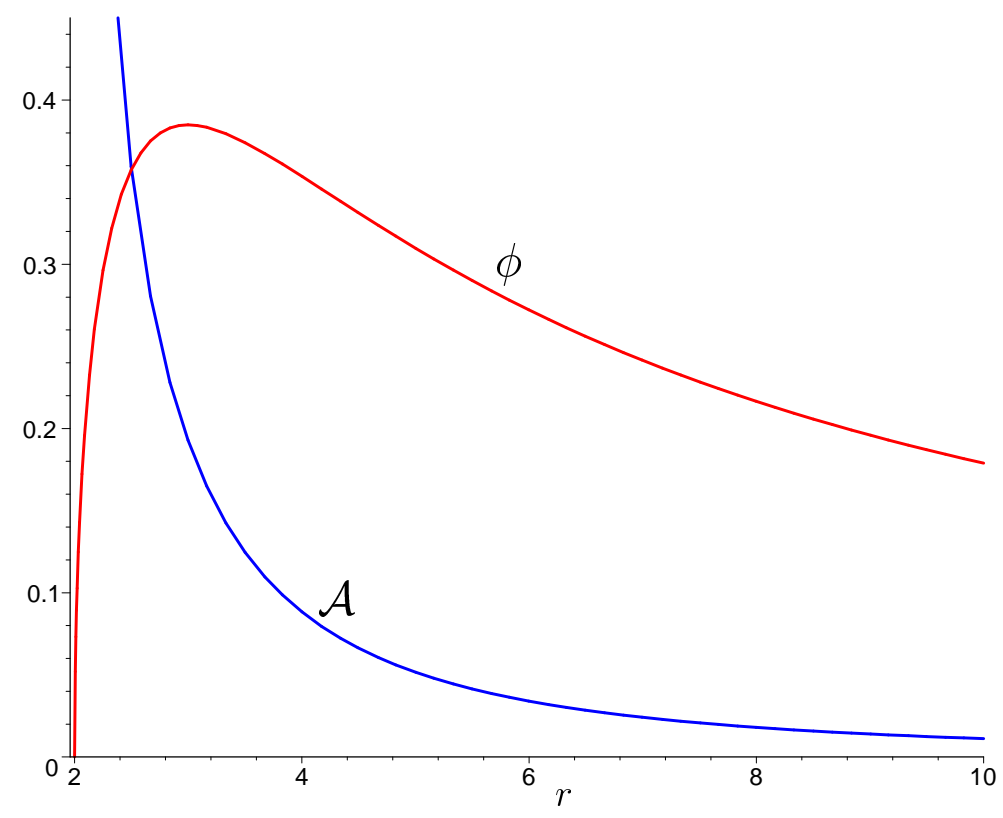

FIG. 1: A plot of $\phi$ and $\mathcal{A}$ with $r$, showing the maxima of $\phi$ at the photon sphere, $r=3 m$. In contrast, $\mathcal{A}$ falls from $\infty$ at the horizon.

are gauge invariant, because they are not first order. Recall the Stewart-Walker lemma 19], which states that if a variable vanishes in the background then it is gauge invariant in the perturbed spacetime.

Equations (40), (55), (36), and (42) are not gauge invariant because zeroth-order terms appear in these equations, i.e., isolated terms involving $\{\mathcal{A}, \mathcal{E}, \phi\}$ not multiplied by a first-order quantity. We therefore introduce the set of gauge-invariant variables

$$
\begin{aligned}
X_{a} & =\delta_{a} \mathcal{E}, \\
Y_{a} & =\delta_{a} \phi, \\
Z_{a} & =\delta_{a} \mathcal{A},
\end{aligned}
$$

which do vanish in the background $(\{\mathcal{A}, \mathcal{E}, \phi\}$ do not vary over a sphere in the background), and so are gauge invariant, by the Stewart-Walker lemma 19]. It is important to notice that we lose no true degrees of freedom in the solutions to the equations by introducing these variables, since we only eliminate possible spherically symmetric perturbations (for which $X_{a}, Y_{a}$ and $Z_{a}$ are automatically zero), but we know from Birkhoff's theorem that all spherically symmetric vacuum spacetimes are Schwarzschild, and therefore any such nontrivial solution must be purely a result of the freedom to chose the frame vectors in the Schwarzchild background.

We may directly obtain the following evolution and propagation equations for these new gauge-invariant variables:

$$
\begin{aligned}
\dot{X}_{a} & =\frac{3}{2} \phi \mathcal{E}\left(\alpha_{a}+\Sigma_{a}-\varepsilon_{a b} \Omega^{b}\right)+\frac{3}{2} \mathcal{E}\left(\delta_{a} \Sigma-\frac{2}{3} \delta_{a} \theta\right)+\varepsilon_{b c} \delta_{a} \delta^{b} \mathcal{H}^{c}, \\
\dot{Y}_{a} & =\left(\frac{1}{2} \phi^{2}+\mathcal{E}\right)\left(\alpha_{a}+\Sigma_{a}-\varepsilon_{a b} \Omega^{b}\right)+\left(\frac{1}{2} \phi-\mathcal{A}\right)\left(\delta_{a} \Sigma-\frac{2}{3} \delta_{a} \theta\right)+\delta_{a} \delta_{c} \alpha^{c}, \\
\hat{X}_{a} & =-2 \phi X_{a}-\frac{3}{2} \mathcal{E} Y_{a}+\frac{3}{2} \phi \mathcal{E} a_{a}-\delta_{a} \delta_{b} \mathcal{E}^{b} \\
\hat{Y}_{a} & =-X_{a}-\frac{3}{2} \phi Y_{a}+\left(\frac{1}{2} \phi^{2}+\mathcal{E}\right) a_{a}+\delta_{a} \delta_{b} a^{b}, \\
\hat{Z}_{a} & =-\left(\frac{3}{2} \phi+2 \mathcal{A}\right) Z_{a}-\mathcal{A} Y_{a}+\mathcal{A}(\phi+\mathcal{A}) a_{a}+\delta_{a} \dot{\theta}-\delta_{a} \delta_{b} \mathcal{A}^{b} .
\end{aligned}
$$

Note that there is not an equation for $\dot{Z}_{a}$ because there is no equation for $\dot{\mathcal{A}}$.

We also find the following constraints by applying the commutator (33) to our new variables:

$$
\begin{aligned}
\varepsilon_{a b} \delta^{a} X^{b} & =3 \phi \mathcal{E} \xi, \\
\varepsilon_{a b} \delta^{a} Y^{b} & =\left(\phi^{2}+2 \mathcal{E}\right) \xi, \\
\varepsilon_{a b} \delta^{a} Z^{b} & =2 \mathcal{A}(\phi+\mathcal{A}) \xi .
\end{aligned}
$$

Equations (78) and (79) replace our equations for (56) and (52) respectively. Similarly Eq. (80) replaces Eq. (42), Eq. (81) replaces (36), and (82) replaces (40). The constraint (85) allows us to derive a pseudo-evolution equation for 
$Z^{a}$ (but we shall not require it). We may also replace our $\dot{\Sigma}$ equation (55) with

$$
\delta_{a} \dot{\Sigma}-\frac{2}{3} \delta_{a} \dot{\theta}=-X_{a}-\mathcal{A} Y_{a}-\phi Z_{a}-\delta_{a} \delta_{b} \mathcal{A}^{b} .
$$

All equations are now gauge invariant. This means that when we have chosen our frame $u^{a}, n^{a}$ in a unique way, then all quantities appearing in the equations are uniquely defined covariant and gauge-invariant quantities, with a physical or geometrical meaning.

\section{Commutation relations for first-order variables}

Having discussed which variables are zeroth order and which are first order, it will be useful to present the following commutation relations for the derivatives of first-order scalars, vectors and tensors. For any scalar, vector or tensor, $\Psi$ :

$$
\begin{aligned}
\dot{\hat{\Psi}}-\hat{\dot{\Psi}} & =\mathcal{A} \dot{\Psi}, \\
\delta_{a} \dot{\Psi}-\left(\delta_{a} \Psi\right)^{\cdot} & =0, \\
\delta_{a} \hat{\Psi}-\widehat{\left(\delta_{a} \Psi\right)} & =\frac{1}{2} \phi \delta_{a} \Psi ;
\end{aligned}
$$

while for a scalar

$$
\delta_{[a} \delta_{b]} \Psi=0,
$$

and for a vector

$$
\delta_{[a} \delta_{b]} \Psi_{c}=\left(\frac{1}{4} \phi^{2}-\mathcal{E}\right) N_{c[a} \Psi_{b]}
$$

and a tensor

$$
\delta_{[a} \delta_{b]} \Psi_{c d}=\frac{1}{2}\left(\frac{1}{2} \phi^{2}-\mathcal{E}\right)\left[N_{c[a} \Psi_{b] d}+N_{d[a} \Psi_{b] c}\right] .
$$

\section{E. Spherical harmonics}

As the equations stand we can't find solutions because of the appearance of angular derivatives ' $\delta_{a}$ '. An appropriate choice of basis functions will allow us to write all first-order variables as an infinite sum over these basis functions, and allow us to replace angular derivatives by a harmonic coefficient. Clearly the spherical symmetry of the background begs us to use spherical harmonics as our basis functions, so here we will develop these appropriately for our formalism. We define our harmonics by analogy with the FLRW case [1], and we refer to [20] for details of other approaches.

Note that all functions and relations below are defined in the background only; we only expand first-order variables, so zeroth-order equations are sufficient.

We introduce spherical harmonic functions $Q=Q^{(\ell, m)}$, with $m=-\ell, \cdots, \ell$, defined on the background, such that

$$
\delta^{2} Q=-\ell(\ell+1) r^{-2} Q, \quad \hat{Q}=0=\dot{Q} .
$$

The function $r$ is covariantly defined by

$$
\phi=2 \frac{\hat{r}}{r}, \quad \dot{r}=0=\delta_{a} r .
$$

This factor is included in our definition (93) so that the equation propagates; it is trivial to show that it evolves also. We have defined $r$ so far up to an arbitrary constant, which reflects our freedom in choosing a particular normalisation of the spherical harmonic functions; we will find it most useful for our purposes to fix this freedom by covariantly defining

$$
r \equiv\left(\frac{1}{4} \phi^{2}-\mathcal{E}\right)^{-1 / 2},
$$

i.e., we identify $r$ defined here with the parameter defined by Eq. (74). We can now expand any first order scalar $\Psi$ in terms of these functions as

$$
\Psi=\sum_{\ell=0}^{\infty} \sum_{m=-\ell}^{m=\ell} \Psi_{\mathrm{S}}^{(\ell, m)} Q^{(\ell, m)}=\Psi_{\mathrm{S}} Q
$$


where the sum over $\ell$ and $m$ is implicit in the last equality. The $\mathbf{S}$ subscript reminds us that $\Psi$ is a scalar, and that a spherical harmonic expansion has been made. Due to the spherical symmetry of the background, $m$ never appears in any equation; we will just ignore it from now on.

We also need to expand vectors and tensors in spherical harmonics. We therefore define the even (electric) parity vector spherical harmonics for $\ell \geq 1$ as

$$
Q_{a}^{(\ell)}=r \delta_{a} Q^{(\ell)} \quad \Rightarrow \quad \hat{Q}_{a}=0=\dot{Q}_{a}, \quad \delta^{2} Q_{a}=(1-\ell(\ell+1)) r^{-2} Q_{a} ;
$$

where the $(\ell)$ superscript is implicit, and we define odd (magnetic) parity vector spherical harmonics as

$$
\bar{Q}_{a}^{(\ell)}=r \varepsilon_{a b} \delta^{b} Q^{(\ell)} \quad \Rightarrow \quad \hat{\bar{Q}}_{a}=0=\dot{\bar{Q}}_{a}, \quad \delta^{2} \bar{Q}_{a}=(1-\ell(\ell+1)) r^{-2} \bar{Q}_{a} .
$$

Note that $\bar{Q}_{a}=\varepsilon_{a b} Q^{b} \Leftrightarrow Q_{a}=-\varepsilon_{a b} \bar{Q}^{b}$, so that $\varepsilon_{a b}$ is a parity operator. The crucial difference between these two types of vector spherical harmonics is that $\bar{Q}_{a}$ is solenoidal, so

$$
\delta^{a} \bar{Q}_{a}=0, \quad \text { while } \quad \delta^{a} Q_{a}=-\ell(\ell+1) r^{-1} Q .
$$

Note also that

$$
\varepsilon_{a b} \delta^{a} Q^{b}=0, \quad \text { and } \quad \varepsilon_{a b} \delta^{a} \bar{Q}^{b}=\ell(\ell+1) r^{-1} Q .
$$

The harmonics are orthogonal: $Q^{a} \bar{Q}_{a}=0$ (for each $\ell$ ), which implies that any first-order vector $\Psi_{a}$ can now be written

$$
\Psi_{a}=\sum_{\ell=1}^{\infty} \Psi_{\vee}^{(\ell)} Q_{a}^{(\ell)}+\bar{\Psi}_{\vee}^{(\ell)} \bar{Q}_{a}^{(\ell)}=\Psi_{\vee} Q_{a}+\bar{\Psi}_{\vee} \bar{Q}_{a}
$$

Again, we implicitly assume a sum over $\ell$ in the last equality, and the $\mathrm{V}$ reminds us that $\Psi^{a}$ is a vector expanded in spherical harmonics.

Similarly we define even and odd tensor spherical harmonics for $\ell \geq 2$ as

$$
\begin{aligned}
& Q_{a b}=r^{2} \delta_{\{a} \delta_{b\}} Q, \quad \Rightarrow \quad \hat{Q}_{a b}=0=\dot{Q}_{a b}, \quad \delta^{2} Q_{a b}=\left[\phi^{2}-3 \mathcal{E}-\ell(\ell+1) r^{-2}\right] Q_{a b} \\
& \bar{Q}_{a b}=r^{2} \varepsilon_{c\{a} \delta^{c} \delta_{b\}} Q, \quad \Rightarrow \quad \hat{\bar{Q}}_{a b}=0=\overline{\bar{Q}}_{a b}, \quad \delta^{2} \bar{Q}_{a b}=\left[\phi^{2}-3 \mathcal{E}-\ell(\ell+1) r^{-2}\right] \bar{Q}_{a b},
\end{aligned}
$$

which are orthogonal: $Q_{a b} \bar{Q}^{a b}=0$, and are parity inversions of one another: $Q_{a b}=-\varepsilon_{c\{a} \bar{Q}_{b\}}{ }^{c} \Leftrightarrow \bar{Q}_{a b}=\varepsilon_{c\{a} Q_{b\}}{ }^{c}$. Any first-order tensor may be expanded

$$
\Psi_{a b}=\sum_{\ell=2}^{\infty} \Psi_{\mathrm{T}}^{(\ell)} Q_{a b}^{(\ell)}+\bar{\Psi}_{\mathrm{\top}}^{(\ell)} \bar{Q}_{a b}^{(\ell)}=\Psi_{\mathrm{T}} Q_{a b}+\bar{\Psi}_{\mathrm{T}} \bar{Q}_{a b} .
$$

We will not write all the equations expanded in spherical harmonics; instead we list here all the replacements which must be made for scalars, vectors and tensors. Note that sums over $\ell$ and $m$ are implicit in these equations below. For brevity, we will sometimes use the aliases

$$
L=\ell(\ell+1), \quad l=(\ell-1)(\ell+2)=L-2 .
$$

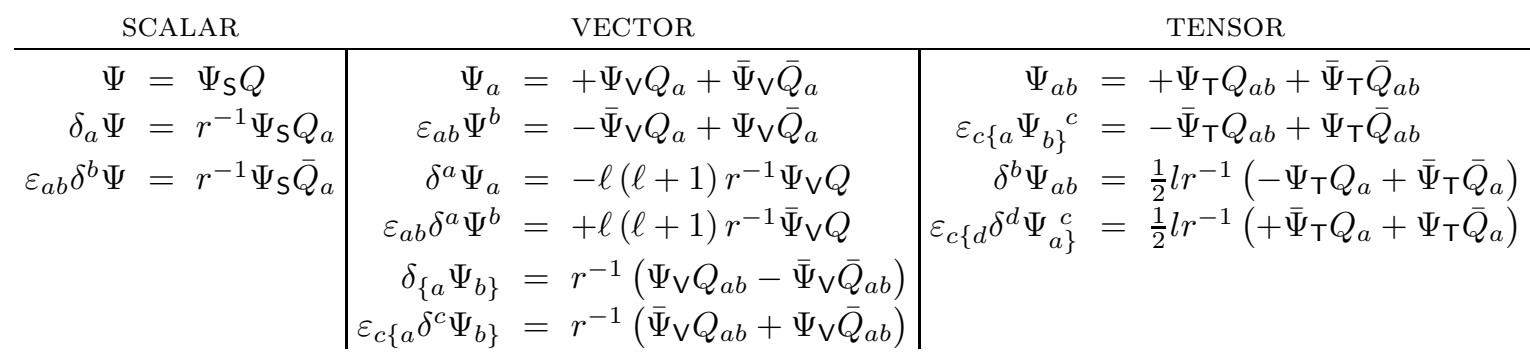

Each vector and tensor equation produces two harmonics equations for each $\ell$, one of odd parity and one of even parity, due to the orthogonality of the vector and tensor harmonics.

We can use these harmonic relations to derive various properties of the $\delta_{a}$ derivative. For example, an important relation we use in deriving the Regge-Wheeler equation in Sec. IV is

$$
2 \delta_{\{a} \delta^{c} \Psi_{b\} c}-\delta^{2} \Psi_{a b}=\left(\mathcal{E}-\frac{1}{2} \phi^{2}\right) \Psi_{a b} .
$$




\section{F. Odd and even parity perturbations}

After decomposing the equations into their harmonic components, we find that they split into two independent subsets, which we refer to as even and odd (parity) perturbations, but are also known as polar and axial perturbations. This splitting is analogous to the well known splitting of scalar, vector and tensor modes in the cosmological situation. The two independent sets of equations utilise the variables

$$
\begin{array}{cc}
\text { Odd parity: } & \mathbf{V}_{\mathrm{O}} \equiv\left(\left\{\overline{\mathcal{E}}_{\mathrm{T}}, \mathcal{H}_{\mathrm{T}}, \bar{\Sigma}_{\mathrm{T}}, \bar{\zeta}_{\mathrm{T}}\right\},\left\{\overline{\mathcal{E}}_{\mathrm{V}}, \mathcal{H}_{\mathrm{V}}, \bar{\Sigma}_{\mathrm{V}}, \Omega_{\mathrm{V}}, \overline{\mathcal{A}}_{\mathrm{V}}, \bar{\alpha}_{\mathrm{V}}, \bar{a}_{\mathrm{V}}, \bar{X}_{\mathrm{V}}, \bar{Y}_{\mathrm{V}}, \bar{Z}_{\mathrm{V}}\right\},\left\{\mathcal{H}_{\mathrm{S}}, \Omega_{\mathrm{S}}, \xi_{\mathrm{S}}\right\}\right)(10 \\
\text { Even parity: } & \mathbf{V}_{\mathrm{E}} \equiv\left(\left\{\mathcal{E}_{\mathrm{T}}, \overline{\mathcal{H}}_{\mathrm{T}}, \Sigma_{\mathrm{T}}, \zeta_{\mathrm{T}}\right\},\left\{\mathcal{E}_{\mathrm{V}}, \overline{\mathcal{H}}_{\mathrm{V}}, \Sigma_{\mathrm{V}}, \bar{\Omega}_{\mathrm{V}}, \mathcal{A}_{\mathrm{V}}, \alpha_{\mathrm{V}}, a_{\mathrm{V}}, X_{\mathrm{V}}, Y_{\mathrm{V}}, Z_{\mathrm{V}}\right\},\left\{\Sigma_{\mathrm{S}}, \theta_{\mathrm{S}}\right\}\right), \quad(10)
\end{array}
$$

where we have defined the vectors $\mathbf{V}_{\mathrm{O}}, \mathbf{V}_{\mathrm{E}}$ for later convenience. The 'parity switching' which occurs between the sets of variables (e.g., $\mathcal{H}_{\top}$ appears in the odd parity system) may be seen in the covariant tensor equations: $\mathcal{H}_{a b}$ always appears alongside a ' $\varepsilon_{a b}$ ' factor relative to other variables such as $\mathcal{E}_{a b}$; similarly for $\Omega^{a}$; it's always seen as $\varepsilon_{a b} \Omega^{b}$, relative to $\Sigma^{a}$, say.

Hereafter, we will assume all equations have been decomposed into their spherical harmonic components, unless stated otherwise, and when we refer to specific equations that are given in tensor form (such as the evolution equations above), we will generally assume too that this has been decomposed implicitly.

\section{G. Time harmonics}

Because the background is static, we can, if we wish, decompose time derivatives of first order quantities into their Fourier components. This is simply assuming an $e^{i \omega \tau}$ time dependence for the first order variables, but we shall make it a bit more precise. Define the time harmonic functions $T^{(\omega)}$ in the background by

$$
\dot{T}^{(\omega)}=i \omega T^{(\omega)}, \quad \hat{T}^{(\omega)}=0=\delta_{a} T^{(\omega)} ; \quad \dot{\omega}=0=\delta_{a} \omega
$$

In the background, this must satisfy

$$
\hat{\dot{T}}+\mathcal{A} \dot{T}=0
$$

which implies

$$
\hat{\omega}=-\mathcal{A} \omega
$$

We may integrate this in the background, in terms of the parameter $x$, or $r$, to give

$$
\omega=\sigma \operatorname{coth} x=\sigma\left(1-\frac{2 m}{r}\right)^{-1 / 2}=\frac{2 \sigma}{\phi r},
$$

where $\sigma$ is a constant. Then any first order variable $\Psi$ (which will usually be an even or odd parity variable discussed above, but it could be any first order scalar, vector or tensor) as

$$
\Psi=\sum_{\omega} \Psi^{(\omega)} T^{(\omega)}=\Psi^{(\omega)} T^{(\omega)}
$$

where the summation is understood in the last equality (which may be an integral, and depends on the types of differential equations and their boundary conditions occurring in the solutions). We can simply replace a dot by $i \omega$ in the equations, as no confusion should occur.

\section{THE REGGE-WHEELER TENSOR AND THE REGGE-WHEELER AND ZERILLI EQUATIONS}

We have presented the full, covariant, gauge-invariant linearised equations for the propagation of gravitational radiation in a perturbed Schwarzschild black hole spacetime, and we have discussed the introduction of spherical harmonics, enabling us to replace $\delta$-derivatives with spherical harmonic indices, as well as remove the tensorial nature of the equations. We could now introduce a perturbed metric and calculate all variables in terms of the metric functions, to show how the standard Regge-Wheeler and Zerilli equations of black hole perturbation theory may be related to this approach, linking all of our variables to these Regge-Wheeler and Zerilli functions. We need not bother 
however: it is possible to find generalisations of these functions and correlations directly, an important test of our theory.

We show in this section that it is possible to find a gauge- and frame-invariant, transverse-traceless tensor that satisfies a closed, covariant, gauge-invariant wave equation. We call this tensor the Regge-Wheeler tensor. Moreover, we demonstrate that, once decomposed into spherical harmonics, and with the appropriate radial coordinate, this equation is the Regge-Wheeler equation for both parities, thus unifying both parities in one TT tensor. We present similar results for the Zerilli equation that normally describes even parity solutions, but defer the derivation of the Zerilli tensor until later in Sec. $\nabla \mathrm{C}$

\section{A. Regge-Wheeler}

We know that gravitational waves propagate in the perturbed Schwarzschild spacetime, and therefore that the TT tensors must satisfy (covariant) wave equations of some sort [see Eqs. (152) and (153) in section VB], as our solutions must recover the plane wave case when $m=0$. When we investigate these wave equations for $\mathcal{E}_{a b}$ and $\mathcal{H}_{a b}$, say, we find that they are not closed: that is, they contain forcing terms from other $1+1+2$ tensors, a feature not present for plane waves. This makes their interpretation and solution nontrivial. In fact, when we look at the second-order wave equations obtained from the covariant equations for any of the $1+1+2$ tensors we find the same story. Can we find some combination of the basic tensors that satisfies a closed, covariant wave equation? Indeed we can, and we outline its derivation here. The formulae are rather formidable, and so we omit a detailed derivation.

In addition to the obvious $1+1+2$ transverse-traceless tensors $\left(\mathcal{E}_{a b}, \mathcal{H}_{a b}, \Sigma_{a b}\right.$ and $\left.\zeta_{a b}\right)$ it is possible to construct many TT tensors from $\delta$-derivatives of vectors and scalars, such as $\delta_{\{a} X_{b\}}, \delta_{\{a} a_{b\}}$, or even $\delta_{\{a} \delta_{b\}} \Omega$, for example. Using the dot and hat equations, along with the commutators, we can obtain the wave equations satisfied by any of these tensors, by calculating the wave operator $\ddot{\Psi}_{\{a b\}}-\hat{\hat{\Psi}}_{\{a b\}}$ for that tensor $\Psi_{a b}$.

If we calculate the wave operators for $\zeta_{a b}$ and $\delta_{\{a} X_{b\}}$ we notice that they contain similar terms in $a_{a}, \alpha_{a}$ and $\xi$. In fact, if in $\ddot{\zeta}_{\{a b\}}-\hat{\hat{\zeta}}_{\{a b\}}$ we substitute for $a_{a}$ from Eq. (80), $\alpha_{a}$ from Eq. (78), and for $\xi$ from Eq. (83) we find, amazingly, that all variables other than $\delta_{\{a} X_{b\}}$ and $\zeta_{a b}$ cancel, leaving the closed wave equation (116) below.

So, we define the dimensionless, gauge-invariant, frame-invariant, transverse-traceless tensor

$$
W_{a b}=\frac{1}{2} \phi r^{2} \zeta_{a b}-\frac{1}{3} r^{2} \mathcal{E}^{-1} \delta_{\{a} X_{b\}}
$$

which obeys the rather nice wave equation

$$
\ddot{W}_{\{a b\}}-\hat{\hat{W}}_{\{a b\}}-\mathcal{A} \hat{W}_{\{a b\}}+\phi^{2} W_{a b}-\delta^{2} W_{a b}=0,
$$

where $\delta^{2}=\delta^{a} \delta_{a}$ is the covariant laplacian on the sheets (approximate 2-spheres, in this case).

We can expand Eq. (116) into SH: let $W=\left\{W_{\mathrm{T}}, \bar{W}_{\mathrm{T}}\right\}$, then Eq. (116) becomes

$$
\ddot{W}-\hat{\hat{W}}-\mathcal{A} \hat{W}+\left\{\frac{\ell(\ell+1)}{r^{2}}+3 \mathcal{E}\right\} W=0 .
$$

Note that both the even and odd parity parts of $W_{a b}$ satisfy the same wave equation (117).

It turns out that Eq. (117) is actually the Regge-Wheeler equation [8] in appropriate coordinates, which we now show. Converting to the parameter $r, \rho \rightarrow r$, and then to the 'tortoise' coordinate of Regge and Wheeler

$$
r_{*}=r+2 m \ln \left(\frac{r}{2 m}-1\right)
$$

and then letting

$$
\psi=\psi_{R W}=W
$$

we find that (117) becomes:

$$
\left(\frac{d^{2}}{d r_{*}^{2}}+\sigma^{2}\right) \psi=V \psi
$$

where

$$
V=V_{R W}=\frac{(r-2 m)}{r^{4}}(\ell(\ell+1) r-6 m),
$$

which is the Regge-Wheeler potential. We will thus refer to $W_{a b}$ as the Regge-Wheeler tensor. 


\section{B. Zerilli}

We have shown that $W_{a b}$ satisfies the Regge-Wheeler equation (117) regardless of parity. However, it is well known that even parity perturbations are governed by the Zerilli equation.

For even parity perturbations the variable

$$
\begin{aligned}
\mathcal{Z} & =\frac{1}{6} \phi\left(l-3 \mathcal{E} r^{2}\right)^{-1}\left(3 r \Sigma_{\mathrm{T}}+2 \mathcal{E}^{-1} \overline{\mathcal{H}}_{\mathrm{V}}\right) \\
& =\frac{2}{3} c_{3}^{-1}\left[3 r \phi \Sigma_{\mathrm{T}}-2 \mathcal{A}^{-1} \overline{\mathcal{H}}_{\mathrm{V}}\right]
\end{aligned}
$$

is one of two fundamental variables, and it can be shown to satisfy the Zerilli equation. The wave equation equation for $\mathcal{Z}$ is

$$
\ddot{\mathcal{Z}}-\hat{\mathcal{Z}}-\mathcal{A} \hat{\mathcal{Z}}+\frac{1}{3} r^{-2}\left\{\frac{1}{4} c_{3}+32 c_{3}^{-2}(L+1) l^{2}\right\} \mathcal{Z}=0 .
$$

We have used the abbreviations:

$$
c_{j}=4(L+1)-j r^{2} \phi^{2},
$$

where we keep the freedom in $j$ for later use.

Making the change to the tortoise coordinate, inserting time harmonic functions, and changing to the variable

$$
\psi=\psi_{\mathcal{Z}}=\mathcal{Z}
$$

we find that (124) is in fact the Zerilli equation, Eq. (120), with

$$
V=V_{\mathcal{Z}}=\frac{r-2 m}{r^{4}\{(L-1) r+6 m\}^{2}}\left[(L-1)^{2}\left[r^{3}(L+7)+24 m r^{2}\right]+36 m^{2} r(L-1)+72 m^{3}\right] .
$$

For the even parity perturbations, then, there are two variables which obey wave equations: the Zerilli variable $\mathcal{Z}$, and the Regge-Wheeler variable $W_{\mathrm{T}}$. They are in fact related by

$$
\hat{\mathcal{Z}}=\frac{i \omega}{3 r^{3} \phi \mathcal{A}} W_{\mathrm{T}}+\frac{r^{2} \phi^{2}\left[c_{3}^{2}-8 l\left(c_{3}+L+4\right)\right]+32 L l(L+1)}{24 r^{4} \phi^{2} \mathcal{A} c_{3}} \mathcal{Z}
$$

as may be found by considering considering $\dot{W}_{\mathrm{T}}$, using Eq. 115). Furthermore, it is possible to find an equation of the form

$$
\hat{W}_{\mathrm{T}}=\text { (stuff) } \hat{\mathcal{Z}}+(\text { more stuff }) \mathcal{Z}
$$

by utilising Eqs. (59), (50) and (61), using the time harmonics throughout, which implies that $\mathcal{Z}$ may be written as a function of $W_{\mathrm{T}}$ and its derivatives. This gives

$$
\hat{W}_{\mathrm{T}}=-\frac{r^{2} \phi^{2}\left[c_{3}^{2}-8 l\left(c_{3}+L+4\right)\right]+32 L l(L+1)}{24 r^{4} \phi^{2} \mathcal{A} c_{3}} W_{\mathrm{T}}-\frac{9 \omega^{2} r^{8} \phi^{4} \mathcal{A}^{2}+L^{2} l^{2}}{3 i \omega r^{5} \phi^{3} \mathcal{A}} \mathcal{Z} .
$$

Thus, the wave variables are not independent. Eqs. (128) and (130) are a two dimensional first-order linear system of differential equations, which could replace the second-order Regge-Wheeler and Zerilli equations. In fact, it is instructive to rewrite Eqs. (128) and (130) in matrix form:

$$
\left(\begin{array}{c}
\hat{W}_{\mathrm{T}} \\
\hat{\mathcal{Z}}
\end{array}\right)=\left(\begin{array}{cc}
\beta & A \\
B & -\beta
\end{array}\right)\left(\begin{array}{c}
W_{\mathrm{T}} \\
\mathcal{Z}
\end{array}\right)
$$

where the definitions of $A, B$ and $\beta$ are obvious from above. Notice that the matrix that couples $\hat{W}_{\mathrm{T}}$ and $\hat{\mathcal{Z}}$ is traceless, and that the special quasinormal modes discussed below correspond to $A=0$.

This form of writing the Regge-Wheeler and Zerilli equations, Eq. (131), may be viewed as just a neat way to write two decoupled second order DE's as two coupled first-order ones; however, it also shows that the two decoupled second order DEs, which Eq. (131) may replace, must be representations of the same physical situation [7] (because $W_{\mathrm{T}}$ is a linear combination of $\mathcal{Z}$ and $\hat{\mathcal{Z}}$, and $\mathcal{Z}$ is a linear combination of $W_{\mathrm{T}}$ and $\left.\hat{W}_{\mathrm{T}}\right)$. We can use this DE to find an odd parity Zerilli variable, which satisfies the Zerilli equation (124), quite easily: simply demand that Eq. (130) hold for the odd parity perturbations too, and substitute for $\bar{W}_{\mathrm{T}}$ from Eq. (115), and then from the propagation equations as appropriate. This then gives us $\overline{\mathcal{Z}}$ as a complicated linear combination of $\overline{\mathcal{E}}_{\mathrm{T}}, \bar{\zeta}_{\mathrm{T}}, \bar{X}_{\mathrm{V}}$ and $\bar{Y}_{\mathrm{V}}$, seemingly unrelated to the even parity Zerilli variable, Eq. (123). This is an illusion, however, and it is possible to find a form for $\overline{\mathcal{Z}}$ very similar to Eq. (123), and consequently a Zerilli tensor, but we defer this until later, in Sec. VC] 


\section{Quasinormal modes}

The Regge-Wheeler and Zerilli equations have been studied in some detail over the years, and their solution is a complicated business 21]. The relevant boundary conditions for the two equations are those that represent a GW perturbation which propagates outwards at infinity $\left(r \sim r_{*} \rightarrow \infty\right)$ and inwards to the horizon $\left(r \rightarrow 2 m, r_{*} \rightarrow-\infty\right)-$ that is, there are no GW propagating in from infinity or out of the horizon. The form of the Regge-Wheeler and Zerilli variables corresponding to this are

$$
\psi \sim e^{i \sigma r_{*}} \quad \text { as } \quad r_{*} \rightarrow-\infty \quad \text { and } \psi \sim e^{-i \sigma r_{*}} \quad \text { as } \quad r_{*} \rightarrow+\infty
$$

where $\psi=W$ or $\psi=\mathcal{Z}$; see, e.g., 21]. It turns out that the only solutions to Eq. (120), with potentials (121) or (127) with boundary conditions (132) require discrete values of the frequency parameter $\sigma$, with $\Im(\sigma)>0$; these are referred to as quasinormal frequencies, and the solutions constructed from them as quasinormal modes (QNMs). Because of the $e^{i \omega \tau}$ time dependence, these decay exponentially in time, which corresponds to energy radiated to infinity or the horizon as GW. This damping in time is important as $\psi$ grows exponentially as $r \rightarrow \infty$. In particular, it also means that the spacetime is not flat at spacelike infinity, but it is flat at future null infinity (i.e., along a null ray).

The factor in front of the $\mathcal{Z}$ in Eq. (130) is rather interesting: evaluate (in terms of $r$ say) to find

$$
9 \omega^{2} r^{8} \phi^{4} \mathcal{A}^{2}+L^{2} l^{2}=(12 m \sigma)^{2}+(L l)^{2}
$$

which has roots at

$$
2 m \sigma= \pm \frac{i}{6} \ell(\ell-1)(\ell+1)(\ell+2)
$$

with the '+' root corresponding to the frequency of the 'special (quasi-normal) mode' discussed in 21, 22], which is the only QNM with $\Re(\sigma)=0$. Since the frequency is purely imaginary the special QNM is not oscillatory in time but merely decays exponentially.

The potentials for the Regge-Wheeler and Zerilli equations when converted into Schrödinger form, $V_{R W}$ and $V_{\mathcal{Z}}$, although appearing very different functionally, are actually very similar (see, e.g., 21]), becoming identical as $\ell \rightarrow \infty$, with peaks lying just beyond the photon sphere, $r=3 \mathrm{~m}$; as $\ell \rightarrow \infty$ the peaks approach the photon sphere (so that the solutions $W$ and $\mathcal{Z}$ become identical for $\ell \rightarrow \infty)$. Thus these one dimensional wave equations represent gravitational waves scattering off the photon sphere, with the same reflection and transmission coefficients [].

It is important to note, however, that the Regge-Wheeler and Zerilli equations allow physical solutions with boundary conditions other than (132), and hence with $\Im(\sigma) \leq 0$, but these solutions will represent GW incoming partially from infinity. These are not relevant for GW detection, but are of interest in their own right, as perturbations of bounded regions, say, may involve such waves.

\section{SOLVING THE EQUATIONS}

Before we discuss the solution of our system of equations, it is worthwhile giving an overview of the structure of the equations. We have three distinct types of equations: propagation, evolution, and constraint. The propagation equations are to be considered the key differential equations, while the evolution and constraints may be considered as auxiliary algebraic equations. This is because the structure of the background varies in the radial direction, so the hat-derivative cannot be expanded in harmonic functions, while the dot- and $\delta$-derivatives are perturbation derivatives, as they do not occur in the background equations, and can thus be expanded in harmonics. We can then analyse and solve the large system of equations using matrix methods. This will provide interesting insight into the problem of black hole perturbations, since it allows us, at any radial position from the black hole, to treat the harmonic variables in (108) and (109) as 'coordinates' - i.e., as a particular choice of basis vectors - in an abstract, 33-dimensional vector space $\mathcal{V}_{33}$ : the non-propagation equations then tell us that only fourteen degrees of freedom are present in the evolution and constraint equations ( out how a subset of this this 14-dimensional solution subspace of $\mathcal{V}_{33}$ propagates radially (that is, they provide the differential system for the remaining degrees of freedom, but there are some undetermined variables), without us having to explicitly specify a basis for the 14-dimensional vector subspace of $\mathcal{V}_{33}$.

Assume a spherical harmonic decomposition of all the equations. Let $\mathbf{V}$ denote the 33-dimensional vector (element of $\left.\mathcal{V}_{33}\right)$ with the variables $\mathbf{V}_{\mathrm{O}}=(108)$ and $\mathbf{V}_{\mathrm{E}}=(109)$ as elements; arrange the vector thus:

$$
\mathbf{V}=(\text { Odd variables } \mid \text { Even variables })=\left(\mathbf{V}_{\mathrm{O}}, \mathbf{V}_{\mathrm{E}}\right) \text {. }
$$


Further, introduce the harmonic expansion in time, so that dot derivatives are everywhere replaced by $i \omega$; the evolution equations without a propagation derivative in them then become a set of 18 algebraic equations. The propagation equations are then of the form

$$
\hat{\mathbf{V}}_{28}=\mathbf{P V},
$$

where $\mathbf{V}_{28}$ denotes the vector consisting of the 28 elements of $\mathbf{V}$ which have a propagation equation. Note that there is no propagation equation for $a^{a}$ (indeed, $a^{a}$ only acts as a forcing term in the differential equations - it is completely undetermined), and some variables do not have an individual propagation equation: there are propagation equations for $\Sigma_{a}-\varepsilon_{a b} \Omega^{b}$ and $\Sigma-\frac{2}{3} \theta$, but not for each of these variables separately (and these can't be generated, as the evolution equations and constraints contain the same degeneracy). $\mathbf{P}$ is the $28 \times 33$ propagation matrix, which contains $i \omega$ terms subsuming evolution equations which have hat derivatives in them.

The 18 remaining evolution equations take the form, in matrix notation

$$
\mathbf{E V}=\mathbf{0},
$$

where $\mathbf{E}$ is a $18 \times 33$ matrix. The 9 constraints are

$$
\mathrm{CV}=\mathbf{0},
$$

where $\mathbf{C}$ is a $9 \times 33$ matrix. Recall that the equations decouple into two sets of equations of opposing parity, which is reflected in the structure of the matrices $\mathbf{E}, \mathbf{P}, \mathbf{C}$, which are all in block-diagonal form:

$$
\left(\begin{array}{c|c}
\begin{array}{c}
\text { Odd } \\
\text { parity }
\end{array} & 0 \\
\hline 0 & \begin{array}{l}
\text { Even } \\
\text { parity }
\end{array}
\end{array}\right),
$$

thus dividing $\mathcal{V}_{33}$ into two divorced subspaces. (We will denote the odd parity upper block of the matrices by a subscript $\mathrm{O}$, and the even lower block by E.) This means that we can treat the odd and even subspaces separately. We will find that the odd equations reduce to a third-order system (i.e., a coupled three-dimensional first-order system of differential equations), and the even equations to a fourth-order system, both of which do not close: the odd has two undetermined variables (from the $\Sigma_{a}-\varepsilon_{a b} \Omega^{b}$ degeneracy, and lack of an equation determining $a^{a}$ ), while the even has three (from the $\Sigma_{a}-\varepsilon_{a b} \Omega^{b}$ and $\Sigma-\frac{2}{3} \theta$ degeneracies, as well as the $a^{a}$ business), all of which reflect freedom in choosing our frame.

In principle, we could adjoin the matrix $\mathbf{C}$ to $\mathbf{E}$ giving a total of 27 algebraic relations between the variables, represented by the $27 \times 33$ matrix

$$
\mathbf{F} \equiv\left(\frac{\mathbf{E}}{\mathbf{C}}\right)
$$

with

$$
\mathbf{F V}=\mathbf{0} .
$$

However, only one constraint equation, Eq. 855), is not represented in the evolution equations (as there is no equation for $Z^{a}$ ). With this exception the constraints evolve and propagate consistently, so eight elements of $\mathbf{C}$ do not contain any more information than $\mathbf{E}$ and $\mathbf{P}$. To put this more elegantly, the fact that the constraints evolve consistently implies that eight rows of $\mathbf{C}$ are just linear combinations of the rows of the matrix $\mathbf{E}$, so that the rank of $\mathbf{F}$ is 19 , just one more than that of $\mathbf{E}$. Let $\mathbf{F}_{\mathcal{L}}$ denote the 19 linearly independent rows of $\mathbf{F}$. Formally, this means that the solution vector $\mathbf{V}$ in $\mathcal{V}_{33}$ must lie in the $(33-19=14$-dimensional $)$ null space, $\mathcal{N}_{14}$, of $\mathbf{F}_{\mathcal{L}}$. Since all equations propagate consistently we can think of this solution space, and the propagation equations acting on it, in an abstract way. We see that the propagation equations are in a certain sense independent of the particular variables we choose to represent the solution.

To actually obtain solutions, however, we must reintroduce 'coordinates' in $\mathcal{N}_{14}$. To this end, we use $\mathbf{F}_{\mathcal{L}}$ to eliminate 19 variables, leaving just 14 (it being largely a matter of choice exactly which 14 , provided they span $\mathcal{N}_{14}$ ); let us denote them by $\mathbf{v}$. The remaining variables can then all be expressed in terms of these fourteen 'coordinate variables'. We may therefore write

$$
\mathbf{V}=\mathbf{M v}
$$


where $\mathbf{M}$ is a $33 \times 14$ matrix of the form

$$
\left(\begin{array}{c|ccc}
\longleftarrow & 6 & \longleftarrow & 8 \\
\text { ODD } & \text { EVEN }
\end{array}\right)
$$

Because of the frame freedom evident in the degenerate variables, we will split the vector $\mathbf{v}$ into two parts: $\mathbf{v}=$ $\left(\mathbf{v}_{D}, \mathbf{v}_{F}\right)$, a 'determined' part containing 9 variables which have an individual propagation equation and a 'free' part containing 5 [say, $\left(\Omega_{\mathrm{V}}, \bar{a}_{\mathrm{V}} \mid \bar{\Omega}_{\mathrm{V}}, a_{\mathrm{V}}, \theta_{\mathrm{S}}\right)$ ] which do not. Inserting Eq. (142) into the propagation equation, Eq. (136), will then finish off the solution, resulting in the equation

$$
\hat{\mathbf{v}}_{D}=\mathbf{B} \mathbf{v}_{D}+\mathbf{A} \mathbf{v}_{F},
$$

where $\mathbf{B}$ is a $9 \times 9$ matrix and $\mathbf{A}$ is $9 \times 5$, giving the solution in the form of a nine-dimensional, first-order (nonautonomous) dynamical system for the vector $\mathbf{v}_{D}$, with forcing terms from the five undetermined variables. The solution (142) is then guaranteed to propagate using (144).

From a naive argument based on counting degrees of freedom of our frame vectors we would expect to be able to eliminate five variables through a careful use of all frame freedom: the congruence $u^{a}$ can be changed by boosting to any new frame moving with some (first-order) three-velocity, giving three degrees of freedom; given a $u^{a}$, we may further make any first-order change in $n^{a}$ that preserves $u^{a} n_{a}=0$, giving two degrees of freedom. Indeed, there are five unknowns in Eq (144), corresponding precisely to these five degrees of freedom, which implies that five equations (rows) of Eq. (144) do not represent true dynamical degrees of freedom. We may specify a frame not only by directly specifying $\mathbf{v}_{F}$ (the most obvious being $\mathbf{v}_{F}=0$ ), selecting some 9-dimensional subspace of $\mathcal{N}_{14}$, but by specifying any variable which can somehow be related to an element of $\mathbf{v}_{F}$ through either Eq. (144) or Eq. (142), thus defining that element of $\mathbf{v}_{F}$, provided, of course, this results in a non-zero, self consistent solution. It turns out, in fact, that the only variables we can't specify in this way are $\mathcal{E}_{a b}, \mathcal{H}_{a b}, W_{a b}, \hat{W}_{a b}$ (the latter being equivalent for these purposes to the Zerilli tensor defined later) - these are frame invariant. Below we choose our frame so that 5 elements of $\mathbf{v}_{D}$ are zero, thus placing 5 rows of Eq. (144) into $\mathbf{M}$, and explicitly having our core dynamical system represent the four true dynamical degrees of freedom - a four dimensional subspace of $\mathcal{N}_{14}$.

To summarise:

- there are 28 propagation equations for 33 variables, which suggests that there are $33-28=5$ 'frame' degrees of freedom in the choice of the two basis vectors $u^{a}$ and $n^{a}$;

- once we have used the time-independence of the background to harmonically decompose the evolution equations, these, combined with the original constraint equations, give rise to a total of 19 linearly independent algebraic relations (not all of the 27 evolution and constraint equations - represented by $\mathbf{F}$ - are independent);

- using these algebraic relations to eliminate 19 variables leaves $33-19=14$ variables to be solved for;

- since the contraint and evolution equations propagate consistently (imposing the constraints 'commutes' with the hat derivative) we can be sure that the propagation equations for eliminated variables can be dropped, since they will follow from the propagation equations for the remaining variables;

- we find then that 9 propagation equations (for $\mathbf{v}_{D}$ ) remain (leaving the $14-9=5$ frame degrees of freedom, $\mathbf{v}_{F}$ );

- finally, since we can choose the 5 frame degrees of freedom more or less arbitrarily we really only have $9-5=4$ true dynamical propagation equations, as we would expect (to see this, imagine choosing 5 of the 9 elements of $\mathbf{v}_{D}$ to be anything at all; then the propagation equations for those variables just fix all five elements of $\mathbf{v}_{F}$, so that the only unknowns that remain are the 4 components of $\mathbf{v}_{D}$ for which we have propagation equations.

\section{A. Determining the full solution: Finding v, M and B}

\section{Odd}

a. General Frame If we don't specify a frame choice, and choose our solution vector as, say, $\mathbf{v}_{D_{0}}=$ $\left(\bar{\Sigma}_{\mathrm{T}}, \bar{\zeta}_{\mathrm{T}}, \bar{X}_{\mathrm{V}}, \overline{\mathcal{A}}_{\mathrm{V}}\right)$, then there are two undetermined variables, which we can choose to be $\mathbf{v}_{F_{\mathrm{O}}}=\left(\Omega_{\mathrm{V}}, \bar{a}_{\mathrm{V}}\right)$, as witnessed in the propagation equation for this solution vector:

$$
\hat{\mathbf{v}}_{D}=\mathbf{B}^{\mathrm{g}} \mathbf{v}_{D}+\mathbf{A}_{O} \mathbf{v}_{F}
$$


All other variables are linear combinations of elements of $\mathbf{v}_{D}$, except $\bar{\Sigma}_{\mathrm{V}}$, which depends on $\Omega_{\mathrm{V}}$; nothing depends on $\bar{a}_{\mathrm{V}}$. Thus, $\mathbf{v}_{F}$ represents a frame freedom in the odd-parity variables, and we can specify it at will.

b. Specific Frame Here, we will choose the frame specifically such that $\bar{Y}_{V}=\overline{\mathcal{A}}_{\vee}=0$. This immediately implies that $\xi_{\mathrm{S}}=\Omega_{\mathrm{S}}=\bar{a}_{\mathrm{V}}=\bar{X}_{\mathrm{V}}=\bar{Z}_{\mathrm{V}}=\Omega_{\mathrm{V}}=0$, which is a rather decent simplification. For our reduced basis vector we will choose

$$
\mathbf{v}=\left(\begin{array}{c}
\bar{W}_{\mathrm{\top}} \\
\hat{\bar{W}}_{\mathrm{\top}}
\end{array}\right) ;
$$

i.e., the governing DE will be the Regge-Wheeler equation. The remaining variables are then given by

$$
\mathbf{V}_{\mathrm{O}}=\left(\begin{array}{c}
\overline{\mathcal{E}}_{\mathrm{T}} \\
\mathcal{H}_{\mathrm{T}} \\
\bar{\Sigma}_{\mathrm{T}} \\
\bar{\zeta}_{\mathrm{T}} \\
\overline{\mathcal{E}}_{\mathrm{V}} \\
\mathcal{H}_{\mathrm{V}} \\
\bar{\Sigma}_{\mathrm{V}} \\
\Omega_{\mathrm{V}} \\
\overline{\mathcal{A}}_{\mathrm{V}} \\
\bar{\alpha}_{\mathrm{V}} \\
\bar{a}_{\mathrm{V}} \\
\bar{X}_{\mathrm{V}} \\
\bar{Y}_{\mathrm{V}} \\
\bar{Z}_{\mathrm{V}} \\
\mathcal{H}_{\mathrm{S}} \\
\Omega_{\mathrm{S}} \\
\xi_{\mathrm{S}}
\end{array}\right)=\mathbf{M}_{\mathrm{O}} \mathbf{v}=\left(\begin{array}{cc}
-C / 2 \phi^{2} r^{4} & -2 / \phi r^{2} \\
\left(-c_{-3}+8 r^{2} \omega^{2}+16\right) / 4 i \omega \phi r^{4} & -C / 2 i \omega \phi^{2} r^{4} \\
1 / i \omega r^{2} & 2 / i \omega \phi r^{2} \\
2 / \phi r^{2} & 0 \\
l / \phi r^{3} & 0 \\
0 & -l / i \omega \phi r^{3} \\
-l / i \omega \phi r^{3} & 0 \\
0 & 0 \\
0 & 0 \\
l / i \omega \phi r^{3} & 0 \\
0 & 0 \\
0 & 0 \\
0 & 0 \\
0 & 0 \\
-L l / i \omega \phi r^{4} & 0 \\
0 & 0 \\
0 & 0 \\
\bar{W}_{\mathrm{T}}
\end{array}\right)
$$

where we have used

$$
C=3 \phi^{2} r^{2}-4 .
$$

\section{Even}

a. General Frame If we don't specify a frame choice, and choose our solution vector as, say, $\mathbf{v}_{D_{\mathrm{E}}}=\left(\Sigma_{\mathrm{T}}, \zeta_{\mathrm{T}}, \Sigma_{\mathrm{V}}+\right.$ $\left.\bar{\Omega}_{\mathrm{V}}, a_{\mathrm{V}}, X_{\mathrm{V}}, Y_{\mathrm{V}}\right)$, then there are three undetermined variables, which we can choose to be $\mathbf{v}_{F_{\mathrm{E}}}=\left(a_{\mathrm{V}}, \bar{\Omega}_{\mathrm{V}}, \theta_{\mathrm{S}}\right)$. The underlying propagation equation is then

$$
\hat{\mathbf{v}}_{D}=\mathbf{B}^{\mathrm{g}} \mathbf{v}_{D}+\mathbf{A}_{\mathrm{E}} \mathbf{v}_{F}
$$

All other variables are linear combinations of elements of $\mathbf{v}_{D_{\mathrm{E}}}$, except $\Sigma_{\mathbf{S}}$ which depends on $\theta_{\mathrm{S}}$. Recall that these freedoms arise from the nature of the propagation and evolution equations: only the combinations of variables, $\Sigma_{a}-\varepsilon_{a b} \Omega^{b}$ and $\Sigma-\frac{2}{3} \theta$, are determined by the propagation and evolution equations, and not the individual variables themselves (although they appear in different combinations in other equations); meanwhile, $a^{a}$ is only present on the rhs of the propagation equations. Thus, $a_{\mathrm{V}}, \bar{\Omega}_{\mathrm{V}}$ and $\theta_{\mathrm{S}}$ represent frame freedom in the even-parity variables, and we can specify these at will (or other variables which indirectly fix these).

b. Specific Frame To concur with the odd case above, we will choose here a frame in which $\mathcal{A}_{\vee}=Y_{\mathrm{V}}=Z_{\mathrm{V}}=0$. We choose

$$
\mathbf{v}=\left(\begin{array}{c}
W_{\mathrm{T}} \\
\mathcal{Z}
\end{array}\right),
$$


so that the governing DE will be Eq. (131). Thus, the basis vector in $\mathcal{V}_{33}$ can easily be converted to the ReggeWheeler equation, or the Zerilli equation, depending on ones frame of mind by substituting for $W_{\mathrm{T}}$ or $\mathcal{Z}$ from the rhs of Eq. [131)]. The other variables are linear combinations of the elements of this solution basis vector:

$$
\mathbf{V}_{\mathrm{E}}=\left(\begin{array}{c}
\mathcal{E}_{\mathrm{T}} \\
\overline{\mathcal{H}}_{\mathrm{T}} \\
\Sigma_{\mathrm{T}} \\
\zeta_{\mathrm{T}} \\
\mathcal{E}_{\mathrm{V}} \\
\overline{\mathcal{H}}_{\mathrm{V}} \\
\Sigma_{\mathrm{V}} \\
\bar{\Omega}_{\mathrm{V}} \\
\mathcal{A}_{\mathrm{V}} \\
\alpha_{\mathrm{V}} \\
a_{\mathrm{V}} \\
X_{\mathrm{V}} \\
Y_{\mathrm{V}} \\
Z_{\mathrm{V}} \\
\Sigma_{\mathrm{S}} \\
\theta_{\mathrm{S}}
\end{array}\right)=\mathbf{M}_{\mathrm{E}} \mathbf{V}=\left(\begin{array}{cc}
2\left(12 \mathcal{E}^{2} r^{4}-C l\right) / c_{3} \phi^{2} r^{4} & -\left[12 \omega^{2} \phi^{2} \mathcal{E} r^{6}+C L l\right] / 2 i \omega \phi^{3} r^{5} \\
2 i \omega / \phi r^{2} & c_{3}\left(c_{-3}-8\right) / 8 \phi^{2} r^{3} \\
4 l / i \omega c_{3} r^{2} & -\left[6 \omega^{2} \mathcal{E} r^{4}+L l\right] / \omega^{2} \phi r^{3} \\
2 / \phi r^{2} & 2 L l / i \omega \phi^{2} r^{3} \\
-l / \phi r^{3} & -L l^{2} / i \omega \phi^{2} r^{4} \\
-12 l \mathcal{E} / i \omega c_{3} r & -3 l \mathcal{A} / 2 \omega^{2} r^{2} \\
l / i \omega \phi r^{3} & -L l\left[l c_{3}+6 \mathcal{E}^{2} r^{4}\right] / \omega^{2} \phi^{2} r^{4} \\
0 & 6 L l \mathcal{A}^{2} / \omega^{2} c_{3} \\
0 & 0 \\
l C / i \omega \phi r^{3} c_{3} & -L l C / 4 \omega^{2} \phi^{2} r^{4} \\
0 & 12 L l \mathcal{A} / i \omega c_{3} \\
0 & -3 L l \mathcal{A} / i \omega r^{2} \\
0 & 0 \\
0 & 0 \\
0 & L l \mathcal{A}\left[5 C^{2}-24 C(L-1)-32(3 L-2)\right] / 2 \omega^{2} \phi^{2} r^{3} c_{3} \\
0 & -3 L l \mathcal{A}^{2}(C+8) / \omega^{2} \phi r c_{3}
\end{array}\right)\left(\begin{array}{c}
W_{\mathrm{T}} \\
\mathcal{Z}
\end{array}\right) .
$$

\section{B. Gravity waves}

The plane wave solutions are given by setting $\mathcal{A}=\phi=0$, with $n^{a}$ lying in the direction of propagation (although, if we keep $\phi=2 / r$ - i.e., set $m=0$ - then it represents the same solution, but $n^{a}$ is a radial direction with an arbitrary centre). In this case only the four TT tensors are non-zero, and $\mathcal{E}_{a b}$ and $\mathcal{H}_{a b}$ represent the curvature of plane gravity waves: dynamical tidal forces orthogonal to the direction of propagation. In the BH case they therefore may be thought of as representing the same part of the GW, although with distortion from the BH itself [24]. This is seen in the wave equations which these tensors satisfy which no longer close, and have forcing terms from other quantities (in a general frame):

$$
\begin{aligned}
\ddot{\mathcal{E}}_{\{a b\}}-\hat{\hat{\mathcal{E}}}_{\{a b\}}-\delta^{2} \mathcal{E}_{a b}-(\phi+5 \mathcal{A}) \hat{\mathcal{E}}_{\{a b\}}-\left(\phi^{2}+8 \mathcal{A}^{2}-5 \mathcal{E}\right) \mathcal{E}_{a b}=2(\phi-2 \mathcal{A}) \delta_{\{a} \mathcal{E}_{b\}}+3 \mathcal{E}(\phi-2 \mathcal{A}) \zeta_{a b}, \\
\ddot{\mathcal{H}}_{\{a b\}}-\hat{\mathcal{H}}_{\{a b\}}-\delta^{2} \mathcal{H}_{a b}-\mathcal{A} \hat{\mathcal{H}}_{\{a b\}}-\left(\frac{1}{4} \phi^{2}-2 \mathcal{A}^{2}+\frac{7}{2} \mathcal{E}\right) \mathcal{H}_{a b}=\left(\frac{7}{2} \phi-\mathcal{A}\right) \delta_{\{a} \mathcal{H}_{b\}}-3 \phi \mathcal{E} \varepsilon_{c\{a} \Sigma_{b\}}{ }^{c}
\end{aligned}
$$

Thus, the principle forcing term for $\mathcal{E}_{a b}$ comes from the shear of $n^{a}$, while $\mathcal{H}_{a b}$ is principally forced by the shear of $u^{a}$.

These TT tensors decouple from all the other variables, but not as wave equations, so it is worthwhile giving the differential equations which these satisfy. Let $\mathcal{W}=\left(\mathcal{W}_{\mathrm{O}}, \mathcal{W}_{\mathrm{E}}\right)$ where

$$
\mathcal{W}_{\mathrm{O}}=\left(\begin{array}{c}
\overline{\mathcal{E}}_{\mathrm{T}} \\
\mathcal{H}_{\mathrm{T}}
\end{array}\right), \quad \text { and } \quad \mathcal{W}_{\mathrm{E}}=\left(\begin{array}{c}
\mathcal{E}_{\mathrm{T}} \\
\overline{\mathcal{H}}_{\mathrm{T}}
\end{array}\right) \text {. }
$$

Then $\mathcal{W}$ satisfies the 4-dimensional first order DE

$$
\hat{\mathcal{W}}=\chi^{-1}\left(\begin{array}{cc}
\Upsilon_{\mathrm{O}} & 0 \\
0 & \Upsilon_{\mathrm{E}}
\end{array}\right) \mathcal{W}
$$

where

$$
\begin{aligned}
\chi & =r^{2} \phi\left\{r^{2} \phi^{2}\left[8 L-r^{2}\left(\phi^{2}+256 \omega^{2}\right)\right]-16\right\} \\
\Upsilon_{\mathrm{O}} & =\left(\begin{array}{cc}
-B_{2}-3 r^{4} \phi^{4}\left(r^{2} \phi^{2}+4\right) & B_{1} \\
-B_{1}-4 L l(i \omega)^{-1} r^{2} \phi^{3} & B_{2}
\end{array}\right),
\end{aligned}
$$




$$
\begin{aligned}
& \Upsilon_{\mathrm{E}}=\left(\begin{array}{cc}
-B_{2}-3 r^{4} \phi^{4}\left(r^{2} \phi^{2}+4\right)+480 r^{4} \phi^{2} \omega^{2} & -4\left(B_{1}-240 i \omega^{3} r^{6} \phi^{3}\right) \\
-B_{1} & -B_{2}
\end{array}\right), \quad \text { and } \\
& B_{1}=i \omega r^{2} \phi\left[-r^{4} \phi^{2}\left(9 \phi^{2}+16 \omega^{2}\right)+8 r^{2} \phi^{2}(2 L+1)-16\right] \text {, } \\
& B_{2}=-\frac{3}{2} r^{6} \phi^{6}+6 r^{4} \phi^{2}\left[\phi^{2}(L-1)+64 \omega^{2}\right]+8 r^{2} \phi^{2}(L-1)+32 .
\end{aligned}
$$

Pretty untidy, but the structure is simple. Note that although decoupling these equations will result in second order

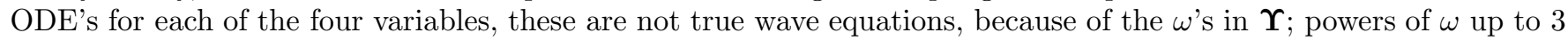
are found in Eq. (155), which correspond to third order dot-derivatives.

In fact, although the Regge-Wheeler tensor, as we have written it, involves $\zeta_{a b}$ which is a kinematical term, it may be written from purely Weyl contributions, using e.g., Eqs. (61) and (50), or just Eq. (51). Therefore, the entire solution may be related relatively simply to the Weyl curvature. Indeed, the variables $\mathcal{E}_{a b}, \mathcal{H}_{a b}, W_{a b}$ are all frame invariant so the relations between these given for the odd and even cases apply regardless of frame; hence, $W_{a b}$ and $\hat{W}_{a b}$ may be given as linear combinations of $\mathcal{E}_{a b}$ and $\mathcal{H}_{a b}$, though not in tensorial form.

\section{The Zerilli tensor}

We have discussed at the end of the section on the Zerilli variable, Sec. IVB how it is possible to find an odd parity Zerilli variable as a linear combination of $\overline{\mathcal{E}}_{\mathrm{T}}, \bar{\zeta}_{\mathrm{T}}$ and $\xi_{\mathrm{S}}$, which was seemingly unrelated to the even parity form of the Zerilli variable. However, we may utilise our solution for the odd parity variables [Eq. (142), although the details of this 'general frame' solution are not given here] in order to write $\overline{\mathcal{Z}}$ as a linear combination of $\bar{\Sigma}_{\mathrm{T}}, \mathcal{H}_{\mathrm{V}}$ and $\mathcal{H}_{\mathrm{S}}$, thus suggesting a connection with the even parity form, Eq. (123):

$$
\left[\frac{1}{9} L^{2} l^{2} \omega^{-2} r^{-8} \phi^{-4} \mathcal{A}^{-2}+1\right] \overline{\mathcal{Z}}=\frac{2}{3} c_{3}^{-1}\left[3 r \phi \bar{\Sigma}_{\mathrm{T}}-2 \mathcal{A}^{-1} \mathcal{H}_{\mathrm{V}}\right]-\frac{1}{9} r^{-3} \phi^{-2} \mathcal{A}^{-2} \mathcal{H}_{\mathrm{S}}
$$

As yet, the similarity with the even case is not quite clear, although nearly there. If we note that the factor multiplying $\overline{\mathcal{Z}}$ on the left hand side is actually a constant (in fact, it is just the special quasi-normal mode factor, discussed earlier), then we can re-define our definition of $\overline{\mathcal{Z}}$, without affecting the differential equation it obeys. Therefore, we define the Zerilli tensor $\mathcal{Z}_{a b}$ by the differential equation:

$$
\left[2-r^{2}\left(2 \mathcal{E}+\delta^{2}\right)\right] \mathcal{Z}_{a b}=\frac{1}{2} r \phi \Sigma_{a b}-\frac{1}{3} r \mathcal{A}^{-1} \varepsilon_{c\{a} \delta^{c} \mathcal{H}_{b\}}-\frac{1}{9} r^{-1} \mathcal{E}^{-2}\left[2-r^{2}\left(2 \mathcal{E}+\delta^{2}\right)\right] \varepsilon_{c\{a} \delta^{c} \delta_{b\}} \mathcal{H},
$$

which is related to our odd and even Zerilli variables by

$$
\begin{aligned}
& \overline{\mathcal{Z}}_{\mathrm{T}}=\left[\frac{1}{9} L^{2} l^{2} \omega^{-2} r^{-8} \phi^{-4} \mathcal{A}^{-2}+1\right] \overline{\mathcal{Z}} \\
& \mathcal{Z}_{\boldsymbol{\top}}=\mathcal{Z}
\end{aligned}
$$

and, of course, both $\overline{\mathcal{Z}}_{\mathrm{T}}$ and $\mathcal{Z}_{\mathrm{T}}$ satisfy the Zerilli equation (124).

The complexity of the definition of this Zerilli tensor (162) is somewhat startling, especially given the simplicity of the analogous Regge-Wheeler tensor. Indeed, the necessity of defining $\mathcal{Z}_{a b}$ via a differential equation really does not inspire confidence in its possible fundamental nature; were it not for the harmonic splitting we are permitted to do due to the nature of the background, we would not be able to actually write down a closed wave equation for $\mathcal{Z}_{a b}-$ the closed tensorial equation it obeys is

$$
\begin{aligned}
& \left(4 \phi^{2} r^{2}-9-r^{2} \delta^{2}\right)\left[\ddot{\mathcal{Z}}_{a b}-\hat{\mathcal{\mathcal { Z }}}_{a b}-\mathcal{A} \hat{\mathcal{Z}}_{a b}\right] \\
& +\frac{16}{3} r^{-2}\left\{\left(\phi^{2} r^{2}-2-r^{2} \delta^{2}\right)^{3}+2\left(\frac{7}{4} \phi^{2} r^{2}-2-r^{2} \delta^{2}\right)\left(\frac{7}{4} \phi^{2} r^{2}-5-r^{2} \delta^{2}\right)^{2}\right\} \mathcal{Z}_{a b}=0 .
\end{aligned}
$$

Hence, only the spherical harmonic amplitudes of $\mathcal{Z}_{a b}, \overline{\mathcal{Z}}_{\mathrm{T}}$ and $\mathcal{Z}_{\mathrm{T}}$, obey a true wave equation.

\section{Discussion}

We have seen in our approach that we may define a TT tensor which satisfies the Regge-Wheeler equation, irrespective of parity, and we have shown that the Zerilli equation may be derived from this tensor in the even case. The transformation equations between the two, Eqs. (128) and (130), then allowed us to derive an odd parity Zerilli variable, and hence a Zerilli tensor. This transformation between parities is made explicit in our approach due to the unification properties of $W_{a b}$ and $\mathcal{Z}_{a b}$. To contrast with the metric approach, the choice of metric functions which 
makes this similarity between the parities explicit is a physically unmotivated expression, which is a complicated linear combination of the metric perturbation functions (see, e.g., Eq. (154) in 7] ). Of our two fundamental tensors, it is fairly clear that the Regge-Wheeler tensor is the most appealing, for two reasons: it is defined in a clear and simple way, and it obeys a covariant wave equation. Recall that it's the harmonic amplitudes of $\mathcal{Z}_{a b}$ which obey wave equations, and not $\mathcal{Z}_{a b}$ itself.

In the frame we have chosen where $\mathcal{A}^{a}=Y^{a}=Z^{a}=0$ we just have $W_{a b}$ governing both the odd and even parity perturbations, which obeys the covariant wave equation (116). The Regge-Wheeler equation describes the dynamically free gravitational field which propagates at the speed of light. It is given here in its fully covariant, gauge- and frame-invariant form. To understand what $W_{a b}$ actually embodies, recall its definition which applies in any frame, Eq. (115). The first term, $\zeta_{a b}$, is just the shear distortion of our sheet (vibrating 2-'spheres') as we move radially along $n^{a}$. The second term is a little more complicated. Recall that in an exact spherically symmetric spacetime, $\mathcal{E}$ is the tidal force measured by our static observers $u^{a}$ in the direction $n^{a}$. There seems to be no reason to change this interpretation in the real perturbed spacetime - here it will just undergo fluctuations, but we can still call it the radial tidal force. 'Radial' loses its meaning in a perturbed spacetime, but if we chose a frame in which $n^{a} \propto \dot{u}^{a}$ (i.e., $\mathcal{A}^{a}=0$ ) an observer could always determine this direction because it would lie precisely in the direction of the external force that they must apply. Spatial fluctuations in the radial tidal forces are characterised by $\left(\mathrm{D}_{a} \mathcal{E}\right) / \mathcal{E}$ which is the comoving fractional gradient of the tidal force; projecting this onto the sheet gives us the gauge invariant (first-order) part of this 3-vector: $\left(\delta_{a} \mathcal{E}\right) / \mathcal{E}=X_{a} / \mathcal{E}$, which tells us the fractional gradient of the radial tidal forces over a sheet - i.e., how the radial tidal forces change from point to point on our vibrating 2-'spheres'. The distortion of this, $\delta_{\{a}\left[\left(\delta_{b\}} \mathcal{E}\right) / \mathcal{E}\right]$, appearing in $W_{a b}$ is the shearing distortion of the radial tidal force gradient. The Regge-Wheeler tensor is thus of a shearing form; it is this tensor which describes GW around a black hole, through the covariant form of the Regge-Wheeler equation (116).

\section{CONCLUSIONS}

We have presented a new perturbation formalism for dealing with systems with spherical symmetry in the background, and we have applied this to the simplest of such systems, the Schwarzschild black hole. Our $1+1+2$ splitting allowed the Schwarzschild solution to be given in covariant form. We then demonstrated that we can derive the usual equations governing perturbations of the spacetime, namely the Regge-Wheeler equation (117), and the Zerilli equation (124), while discussing in detail our new method. We have also shown that there exist Regge-Wheeler and Zerilli tensors which unify the odd and even parity perturbations; indeed, the Regge-Wheeler tensor was shown to satisfy a closed covariant wave equation which governs the dynamics of the whole problem. This sets the basis for future studies of more general astrophysical systems, which only require an appropriate change of the background, for which the possible applications are myriad.

The method has several important aspects. The first is the covariant spacetime splitting itself. In general, the two threading vectors $u^{a}$ and $n^{a}$ may be chosen arbitrarily, defining the sheet on which the vectors and tensors exist (strictly speaking, in general the sheet is not a true surface, but a collection of tangent planes). This makes the approach a halfway house between the $1+3$ approach and the orthonormal tetrad approach, and provides a completion of the covariant formalism. (Recall that a unique tetrad cannot be defined in isotropic or locally rotationally symmetric spacetimes [1, 5], so this is the ideal compromise between the two in such cases.) For systems with spherical symmetry in the background, we have seen that when $n^{a}$ is radial in the background, the perturbed spacetime becomes a tractable problem, because all vectors and tensors become first order, allowing decomposition with suitable harmonic functions - spherical harmonics, in this case. Time harmonics are also introduced to simplify the solution, allowed when the background is static, but these are not strictly necessary, as the dot-derivative is a scalar operator, and can be dealt with by standard techniques. So far this merely writes the equations in an alternative form. At this point finding the solution is relatively trivial: our approach simply requires solving a linear system of algebraic equations, and this is all there is to it. The underlying dynamics are then automatically given by the small system of differential equations that remain - wave equations, if desired, then may be derived (where they exist) by differentiating this equation.

An important physical aspect of our approach is that it uses a set of covariantly defined quantities with genuine physical significance, which makes it clear which objects are crucial for the detection and measurement of gravitational waves. Put simply, GW detectors essentially measure gravitational tidal forces; that is, they are sensitive to the dynamical Weyl curvature, encoded in the electric Weyl tensor, $E_{a b}$, and this dynamical Weyl curvature forces the working parts of any GW detector through the right hand side of the geodesic deviation equation. We have shown that there is a gauge-invariant TT tensor that describes GWs of either parity, and is closely related to the variation of the radial tidal force. Thus it is clear that our formalism is dealing with real, physically measurable, objects from the start. Indeed, we have discussed how a subset of four of all thirty-three variables is sufficient to determine the full 
dynamics of the spacetime.

There are many possible extensions of the work we have presented. The obvious extension is to perturbations of static stars, but we envisage that our method will be widely applicable to many other astrophysical situations, such as systems with a dynamical background - e.g., collapsing stars, type Ia supernovae, etc. - where the scalar background equations have two (non-tensorial) derivatives in them. In the static background case, where we can introduce timeharmonic functions in the perturbed equations, solving the equations is a simple problem of solving a linear system of equations, and one is left with a first order non-autonomous system of ordinary differential equations, whose dimension is small compared to the original system, plus linear relations among the remaining variables in terms of the basis vector of the dynamical system. All the physics of the spacetime is contained in this small dynamical system. With a dynamical background, it may not be as simple as this, but we do not envisage it being much more difficult to find the full solution; one may have to be careful choosing one's observers (perhaps comoving with the matter in the case of a collapsing star). In any event, there is much that can be achieved. In any situation where there is a preferred spacelike vector field present, the covariant $1+1+2$ sheet formalism should provide new insight.

\section{Acknowledgments}

We would very much like to thank Peter Dunsby, George Ellis and Roy Maartens for lots of continuous advice and encouragement on this project. We would also like to thank Gerold Betschart, Bonita de Swardt, Kostas Kokkotas, Nazeem Mustapha and Jorge Pullin for useful discussions and/or comments. CAC was funded by NRF (South Africa), and RKB was funded by EPSRC (UK).

[1] G. F. R. Ellis and H. van Elst, in M. Lachieze-Rey (ed.), Theoretical and Observational Cosmology, NATO Science Series, Kluwer Academic Publishers (1998) gr-qc/9812046v4

[2] Ellis, G.F.R. and Bruni, M. Phys Rev. D 401804 (1989)

[3] Challinor, A.D. and Lasenby, A.N. Phys. Rev. D 58023001 (1998); Astrophys. J. 5131 (1999)

[4] Maartens, R., Gebbie, T. and Ellis, G.F.R. Phys. Rev. D 59083506 (1999)

[5] van Elst, H. and Ellis, G. F. R., Class. Quantum Grav. 131099 (1996); van Elst, H., Ph.D. thesis, University of London (1996)

[6] Barish, B. and Weiss, R., Phys. Today 52, 44-50 (1999); B Willke et al., Class. Quantum Grav. 19, 1377 (2002); M Ando et al., Class. Quantum Grav. 19, 1409 (2002); F Acernese et al., Class. Quantum Grav. 19, 1421 (2002).

[7] Chandrasekhar, S. The Mathematical Theory of Black Holes. Oxford, (1983)

[8] Regge, T. and Wheeler, J.A. Phys. Rev. 1081063 (1957)

[9] Zerilli, F.J. Phys. Rev. Lett. 24737 (1970)

[10] Gerlach, U. H. and Sengupta, U. K., Phys. Rev. D, 192268 (1979); ibid. 221300 (1980)

[11] Gundlach, C. and Martín-García, J.M. $g r-q c / 9906068$ and $g r-q c / 0012056$

[12] Sarbach, O. and Tiglio, M.: Phys. Rev. D 64084016 (2001)

[13] Jezierski, J.: Gen. Rel. Grav. 311855 (1999) $g r-q c / 9801068$

[14] Zafiris, E., J. Math. Phys. 385854 (1997)

[15] Mason, D.P. and Tsamparlis, M., J. Math. Phys. 262881 (1985)

[16] Greenberg, P.J., J. Math. Anal. Applic. 30128 (1970)

[17] Tsamparlis, M. and Mason, D.P., J. Math. Phys. 241577 (1983)

[18] Bruni, M., Matarrese, S., Mollerach, S. and Sonego, S., Class. Quantum Grav. 142585 (1997); Bruni, M. and Sonego, S., Class. Quantum Grav., 16 L29 (1999)

[19] Stewart, J.M. and Walker, M. Proc. R. Soc. London A 43149 (1974)

[20] Thorne, K.S., Rev. Mod. Phys. 52299 (1980)

[21] Nollert, HP. Class. Quantum Grav. 16 R159 (1999); Kokkotas, K.D. and Schmidt, B.G., Living Rev. Relativity, 22 (1999), http//www .livingreviews.org/Articles/Volume2/1999-2kokkotas/

[22] Liu, H. and Mashhoon, Class. Quantum Grav. 13233 (1996)

[23] Nollert, HP. and Schmidt, B.G., Phys. Rev. D 452617 (1992)

[24] Price, R.H. and Thorne, K.S., Phys. Rev. D 33915 (1986) 